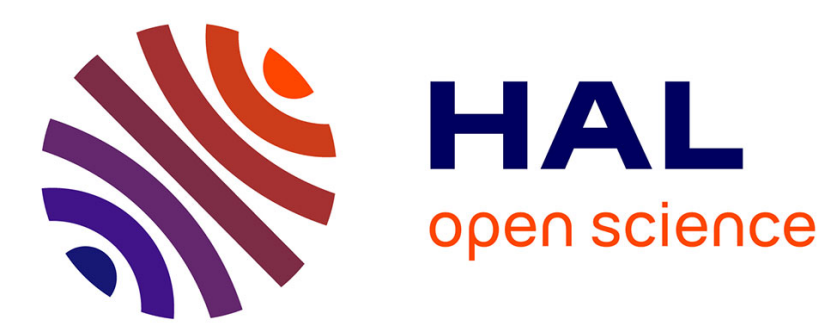

\title{
Chapter VII The Rare Earth Garnets
}

Louis Néel, R. Pauthenet, B. Dreyfus

\section{To cite this version:}

Louis Néel, R. Pauthenet, B. Dreyfus. Chapter VII The Rare Earth Garnets. Progress in Low Temperature Physics, 4, Elsevier, pp.344-383, 1964. hal-02888384

\section{HAL Id: hal-02888384 https://hal.science/hal-02888384}

Submitted on 29 Jul 2020

HAL is a multi-disciplinary open access archive for the deposit and dissemination of scientific research documents, whether they are published or not. The documents may come from teaching and research institutions in France or abroad, or from public or private research centers.
L'archive ouverte pluridisciplinaire HAL, est destinée au dépôt et à la diffusion de documents scientifiques de niveau recherche, publiés ou non, émanant des établissements d'enseignement et de recherche français ou étrangers, des laboratoires publics ou privés. 
CHAPTER VII

\section{THE RARE EARTH GARNETS}

BY

\section{NÉEL, R. PAUTHENET AND B. DREYFUS}

UNIVERsity of Grenoble, France

Contents: 1 . Introduction, 344. - 2. The magnetic properties of the rare earth garnets, 346. - 3. The levels of rare earth ions in the garnets, 369.

\section{Introduction (by L. NÉEL)}

The rare earth garnets, of general formula $\mathrm{Fe}_{5} \mathrm{M}_{3} \mathrm{O}_{12}$ where $\mathrm{M}$ is a trivalent rare earth ion, form an important class of magnetic compounds whose properties can be remarkably simply and quite accurately interpreted by a theory of three ferrimagnetic sub-lattices. Theoretically, their study is of interest because of the large number of atomic substitutions that can be realised in the lattice and one hopes that this may lead to a solution of the problem of magnetic interactions. Their practical interest, especially at high frequencies, is considerable, as they are excellent electrical insulators, preparable as monocrystals and characterised by very narrow resonance lines.

The story of the rare earth garnets started in 1950, at Strasbourg. Forestier and Guiot-Guillain showed ${ }^{1}$ that, on heating an equi-molecular mixedprecipitate of $\mathrm{Fe}_{2} \mathrm{O}_{3}$ and $\mathrm{M}_{2} \mathrm{O}_{3}$ where $\mathrm{M}$ is a rare earth such as $\mathrm{Nd}, \mathrm{Er}$, $\mathrm{Y}, \mathrm{Sm}, \mathrm{Pr}, \mathrm{La}$, they obtained strongly ferromagnetic products which they identified as $\mathrm{Fe} \mathrm{MO}_{3}$ and whose Curie points ranged from 520 to $740^{\circ} \mathrm{K}$. In 1951, Guiot-Guillain ${ }^{2}$ showed that the ferrites of praesodymium and lanthanum had a perovskite-type structure.

Continuing their studies in 1952 and 1953, Forestier and Guiot-Guillain ${ }^{3}$ brought to light a curious fact: with $\mathrm{M}=\mathrm{Yb}, \mathrm{Tm}, \mathrm{Y}, \mathrm{Gd}$ and $\mathrm{Sm}$, the aforementioned products had two Curie-points about a hundred degrees apart. These points varied regularly from one metal to the next as a function of the atomic radius of $\mathrm{M}$. On passing through the upper Curie-point one obtained a strong thermoremanent magnetisation.

These results attracted the attention of the "Laboratoire de Magnétisme" 
at Grenoble: Pauthenet and Blum ${ }^{4}$ prepared a gadolinium ferrite and showed in 1954 that, besides the two Curie-points $\theta_{1}=570^{\circ} \mathrm{K}$ and $\theta_{2}=678^{\circ} \mathrm{K}$ already mentioned, there was a third temperature $\theta_{3}=306^{\circ} \mathrm{K}$ at which the spontaneous magnetisation was zero. It certainly seemed that this was a compensation temperature, in the sense of Néel's theory of ferrimagnetism; that is, a temperature where the magnetisation changes sign.

At the same time, Néel ${ }^{5}$ proposed a likely explanation of these facts on the supposition that the $\mathrm{Fe}^{3+}$ ions in the lattice formed a ferrimagnetic ensemble A having a spontaneous magnetisation opposite in direction to that of the sublattice $\mathrm{B}$ of $\mathrm{M}^{3+}$ ions. The molecular coupling field was sufficiently weak such that for temperatures above that of liquid air the magnetisation of the $B$ ions was proportional to the field. In particular, Néel suggested that the point $\theta_{3}$ corresponded to an exact compensation of the magnetisations of the ferrimagnetic $A$ ions and $B$ ions. A few days later ${ }^{6}$ the existence of such compensation points was confirmed for the ferrites of dysprosium and erbium, at $246^{\circ}$ and $70^{\circ} \mathrm{K}$ respectively; at these temperatures the remanent magnetisation changed sign.

In spite of this success the supposed ferrimagnetic distribution of the $\mathrm{Fe}^{3+}$ ions was inconsistent with the recently confirmed perovskite structure of the compound $\mathrm{FeMO}_{3}$. At the request of L. Néel, Remeika at the Bell Telephone Laboratory prepared monocrystals of $\mathrm{FeMO}_{3}$ which, after study by $\mathbf{S}$. Geller ?, were sent to Grenoble.

Starting from the idea that the observed phenomena were due to a new type of compound, Bertaut and Forrat showed in January $1956^{8}$ that one was dealing with a cubic compound $\mathrm{Fe}_{5} \mathrm{M}_{3} \mathrm{O}_{12}$, space-group $\mathrm{O}_{h}^{10}$ la 3d, with eight molecules per unit cell - an entirely unexpected discovery. The ferrimagnetic distribution of the $\mathrm{Fe}^{3+}$ ions was thus 24 ions on the tetrahedral sites $24 \mathrm{~d}$, oppositely magnetised to the remaining $16 \mathrm{Fe}^{3+}$ ions on the octahedral sites $16 \mathrm{a}$.

In the following months, many experiments quantitatively verified the accuracy of this model. In particular, Pauthenet, after his work on pure $\mathrm{Fe} \mathrm{GdO}_{3}$ and $\mathrm{Fe}_{3} \mathrm{Gd}_{5} \mathrm{O}_{12}$, showed ${ }^{9}$ that gadolinium ferrite (the object of his first investigations ${ }^{4}$ ) was in fact a mixture of $0.26 \mathrm{~g}$ of garnet, $0.64 \mathrm{~g}$ of perovskite and $0.1 \mathrm{~g}$ of $\mathrm{Gd}_{2} \mathrm{O}_{3}$; the points $\theta_{1}$ and $\theta_{2}$ were respectively the Curie-points of the garnet and perovskite. He soon completed his results with a study of the complete series of rare earth garnets ${ }^{10}$, while, with Aléonard en Barbier he studied 11 the properties of the isolated sublattice of $\mathrm{Fe}^{3+}$ ions in yttrium ferrite. All these results were communicated to the Congress of Physics at Moscow ${ }^{12}$ (25-31 May, 1956). 
The same research teams also did the first work on substitutions ${ }^{13}$, the accurate determination of the parameters ${ }^{14}$ and, with Herpin and Meriel ${ }^{15}$, structure determination by neutron diffraction.

Following this, the Bell Telephone Labs. conducted a beautiful series of experiments on substitutions in the garnets, originating in the work of $S$. Geller ${ }^{16}$.

The two following articles are devoted to the garnets, the first by $\mathbf{R}$. Pauthenet on their magnetic properties, and the second by B. Dreyfus on their optical and thermal properties, especially at low temperatures. In view of the numerous articles on the garnets since their discovery, it has been necessary to make a limited choice of the subject treated.

\section{The Magnetic Properties of the Rare Earth Garnets (by R. PAUTHENET)}

The aim of this section is to describe only the more important results obtained from the study of the magnetic properties of the garnet-type ferrites in a static field; in particular, those results useful for the understanding of many interesting problems that these compounds pose.

\subsection{Preparation of the Rare Earth Garnet-Type Ferrites}

There are two methods used for the preparation of the garnet-type ferrites in a polycrystalline form. The first consists of a solid-state diffusion of an intimate compressed mixture of oxides, obtained by heating for eight hours between 1340 and $1380^{\circ} \mathrm{C}$. The second consists of the thermal decomposition of a homogenous co-precipitate of the nitrates at $400^{\circ} \mathrm{C}$ followed by baking at $1200^{\circ} \mathrm{C}$ for several hours ${ }^{8}$. The first method is quicker but the products are a little less pure and homogenous than those obtained by the second. This latter can be improved in detail to obtain very pure compounds ${ }^{17}$. The garnet-type ferrites exist for yttrium and all the rare earths except lanthanum, cerium, praesodymium and neodymium; preparation with prometheum, which is radioactive, has not been attempted; the ionic radius of the rare earth must be less than $1.13 \AA$ (Table 1 ).

It became quickly evident that work with monocrystals was of great interest. Nielsen and Dearborn ${ }^{18}$ perfected a method of preparation using $\mathrm{PbO}$ as a solvent; study of the phase diagram $\mathrm{Fe}_{2} \mathrm{O}_{3}-\mathrm{M}_{2} \mathrm{O}_{3}-\mathrm{PbO}$ showed that a satisfactory initial composition was $44 \mathrm{Fe}_{2} \mathrm{O}_{3}-3.5 \mathrm{M}_{2} \mathrm{O}_{3}-$ $52.5 \mathrm{~Pb}$ O. The method consists in heating the above mixture in a platinum crucible for five hours, cooling at less than $1^{\circ} \mathrm{C}$ per hour to about $900^{\circ} \mathrm{C}$ and finally quenching it. One can thus obtain monocrystals of garnet-type ferrite of about $1 \mathrm{~cm}$ in size mixed with crystals of magnetoplombite, rare 
TABLe 1

\begin{tabular}{|c|c|c|c|c|c|c|c|c|c|c|c|c|c|c|c|c|}
\hline $\mathbf{M}^{3+}$ & $\mathbf{Y}$ & $\mathbf{L a}$ & $\mathrm{Ce}$ & $\mathbf{P r}$ & Nd & $\mathbf{P m}$ & Sm & Eu & Gd & $\mathbf{T b}$ & Dy & Ho & $\mathrm{Er}$ & $\operatorname{Tm}$ & $\mathbf{Y b}$ & $\mathbf{L u}$ \\
\hline $\boldsymbol{L}$ & 0 & 0 & 3 & 5 & 6 & 6 & 5 & 3 & 0 & 3 & 5 & 6 & 6 & 5 & 3 & 0 \\
\hline$s$ & 0 & $\mathbf{0}$ & $\frac{1}{2}$ & $\frac{2}{2}$ & $\frac{3}{2}$ & $\frac{4}{2}$ & $\frac{5}{2}$ & $\frac{6}{2}$ & $\frac{7}{2}$ & $\frac{6}{2}$ & $\frac{5}{2}$ & $\frac{4}{2}$ & $\frac{3}{2}$ & $\frac{2}{2}$ & $\frac{1}{2}$ & 0 \\
\hline$J$ & 0 & $\mathbf{0}$ & $\frac{5}{2}$ & 4 & $\frac{9}{2}$ & 4 & $\frac{5}{2}$ & 0 & $\frac{7}{2}$ & 6 & $\frac{15}{2}$ & 8 & $\frac{15}{2}$ & 6 & $\frac{7}{2}$ & 0 \\
\hline $\begin{array}{l}\text { ionic radius of } \\
\text { Goldschmidt }(\AA)\end{array}$ & 1.06 & 1.22 & 1.18 & 1.16 & 1.15 & & 1.13 & 1.13 & 1.11 & 1.09 & 1.07 & 1.05 & 1.04 & 1.04 & 1.00 & 0.99 \\
\hline$a \pm 0.004 \AA$ & $12.37 \mathrm{~g}$ & & & & & & $12.52_{4}$ & $12.51 \mathrm{~g}$ & $12.46_{5}$ & $12.45_{2}$ & $12.40_{3}$ & $12.38_{1}$ & 12.360 & $12.32_{1}$ & $12.29_{1}$ & 12.277 \\
\hline $\mathbf{J}_{0} \mu_{\mathrm{B}}$ & $\begin{array}{r}9.44 \\
\pm 0.55\end{array}$ & & & & & & $\begin{array}{l}\quad 9.32 \\
\pm 0.3\end{array}$ & $\begin{aligned} & 5.15 \\
\pm & 0.3\end{aligned}$ & $\begin{array}{r}30.3 \\
\pm 0.3\end{array}$ & $\begin{array}{r}31.4 \\
\pm \quad 0.3\end{array}$ & $\begin{array}{r}32.5 \\
\pm 0.3\end{array}$ & $\begin{array}{r}27.5 \\
\pm 0.3\end{array}$ & $\begin{array}{r}23.1 \\
\pm \quad 0.3\end{array}$ & $\begin{array}{l}2.0 \\
\pm 0.1 \pm\end{array}$ & $\begin{array}{l}0 \\
+0.3\end{array}$ & $\begin{array}{r}8.32 \\
\pm 0.05\end{array}$ \\
\hline$\theta_{\mathrm{r}}^{0} \mathbf{K} \pm \mathbf{2}$ & 560 & & & & & & 578 & 566 & 564 & 568 & 563 & 567 & 556 & 549 & 548 & 549 \\
\hline $\boldsymbol{\theta}_{\mathrm{c}}^{\circ} \mathbf{K} \pm \mathbf{2}$ & & & & & & & & & 290 & 246 & 220 & 136 & 84 & $\begin{array}{l}4<\theta_{\mathrm{c}} \\
\theta_{\mathrm{c}}<20\end{array}$ & & \\
\hline $\boldsymbol{\theta}_{\mathbf{p}}{ }^{\circ} \mathbf{K}$ & & & & & & & & & -24 & -8 & -32 & -6 & -8 & & & \\
\hline
\end{tabular}


earth orthoferrites and iron oxide. The crucible used is quickly corroded and Nielsen ${ }^{18}$ has suggested the use of $\mathrm{Pb} \mathrm{F}_{2}$ as a solvent to prevent this; fusion is then lowered to about $1250^{\circ} \mathrm{C}$.

\subsection{Crystal Structure}

The crystallographic study of the rare earth garnet-type ferrites has been done by Bertaut and Forrat ${ }^{8,14}$ and taken up again by Geller and Gilleo ${ }^{20,21}$.

$$
5 F_{2} O_{3} \cdot 3 M_{2} O_{3}
$$

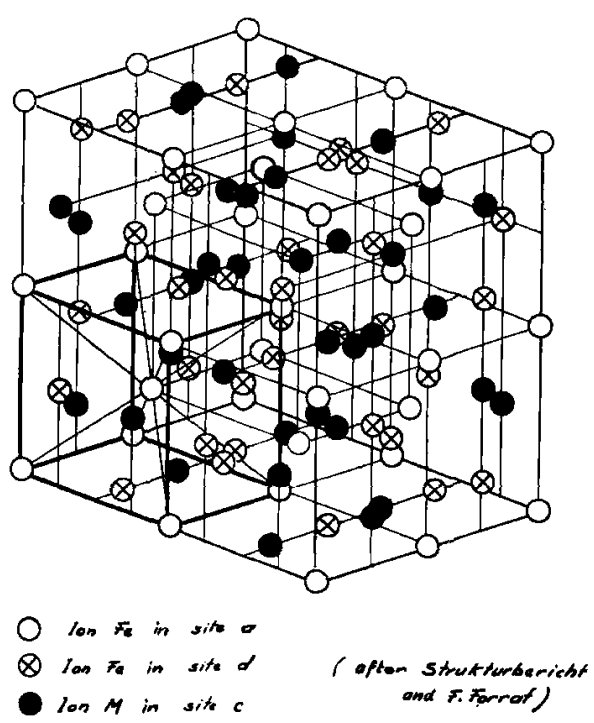

Fig. 1. Positions of the magnetic ions in the garnet structure.

These ferrites are cubic in structure and are isomorphous with the garnet $\mathrm{Ca}_{3} \mathrm{Fe}\left(\mathrm{Si} \mathrm{O}_{4}\right)_{3}$ (Fig. 1). There are eight units of $\mathrm{Fe}_{5} \mathrm{M}_{3} \mathrm{O}_{12}$ in the unit cell. The oxygen ions define three types of sites at which the magnetic ions are found; $16 \mathrm{Fe}^{3+}$ ions occupy the 16 a positions which are at the centre of an octahedral arrangement of oxygen ions (Fig. 2a);24 Fe ${ }^{3+}$ ions occupy the positions $24 \mathrm{~d}$ at the centre of a tetrahedron (Fig. 2b); finally the $24 \mathrm{M}^{3+}$ ions occupy the positions $24 \mathrm{c}$ inside a polyhedron with eight vertices and twelve edges, which can be appropriately represented as an irregular hexahedron obtained on distorting a cube by torsion about a tetrad axis followed by a slight distortion of the faces (Fig. 2c). The totality of the a-sites 
defines the sub-lattice (a); similarily we have the sub-lattices (d) and (c). $\mathrm{A} \mathrm{Fe}^{3+}$ ion in a 16 a position will be written $\mathrm{Fe}^{3+}$ (a); its position in the unit cell is $\left(0,0, \frac{1}{2}\right)$; the $\mathrm{Fe}^{3+}$ (d) ions are at $\left(0, \frac{1}{4}, \frac{3}{8}\right)$ and the $\mathrm{M}^{3+}$ (c) ions at $\left(\frac{1}{4}, \frac{1}{8}, \frac{1}{2}\right)$ and $\left(0, \frac{1}{4}, \frac{5}{8}\right)$; the $96 \mathrm{~h}$ oxygen sites are defined by $x=-0.027_{4}$, $y=0.057_{2}, z=0.149_{2}$ and are given by

$$
\begin{array}{cccc} 
\pm x y z ; \quad x \tilde{y}\left(\frac{1}{2}-z\right) ; & \left(\frac{1}{2}-x\right) y \bar{z} ; \quad \bar{x}\left(\frac{1}{2}-y\right) z ; & \left(\frac{1}{4}-y\right)\left(\frac{1}{4}-x\right)\left(\frac{1}{4}-z\right) ; \\
\left(\frac{1}{4}+y\right)\left(\frac{1}{4}-x\right)\left(\frac{3}{4}+z\right) ; & \left(\frac{1}{4}-y\right)\left(\frac{3}{4}+x\right)\left(\frac{1}{4}+z\right) ; & \left(\frac{3}{4}+y\right)\left(\frac{1}{4}+x\right)\left(\frac{1}{4}-z\right) .
\end{array}
$$

The crystal parameter $a$ (Table 1 ) decreases regularly with the ionic radius of the rare earth, in agreement with the rule for lanthanide contraction, from $12.52_{4} \AA$ for $5 \mathrm{Fe}_{2} \mathrm{O}_{3} \cdot 3 \mathrm{Sm}_{2} \mathrm{O}_{3}$ to $12.27_{7}$ for $5 \mathrm{Fe}_{2} \mathrm{O}_{3} \cdot 3 \mathrm{Lu}_{2} \mathrm{O}_{3}$. To discuss the magnitude and sign of the magnetic interactions it is useful to know the inter-ionic distances between nearest neighbours and the bond angles between neighbouring magnetic ions and the intermediate oxygen ion; these are given in Table 2; yttrium ferrite furnishes a typical order of magnitude for the distances.

\subsection{Magnetostatic Properties}

\subsubsection{Experimental Results}

Experiment shows that for temperatures less than about $550^{\circ} \mathrm{K}$ and for fields between 5000 and $20000 \mathrm{Oe}$, the magnetisation $J$ of the ferrites of yttrium, samarium, europium and lutetium varies with the field according to the law of approach to saturation

$$
J=J_{\mathbf{s}}(1-a / H) ;
$$

from this we can find the spontaneous magnetisation $J_{\mathrm{s}}$ by extrapolation. For the ferrites of gadolinium, terbium, dysprosium, holmium and erbium

(a)

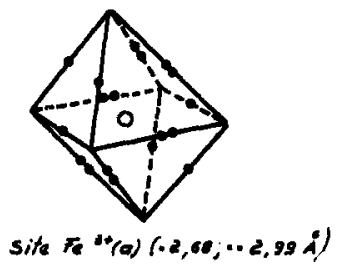

(b)

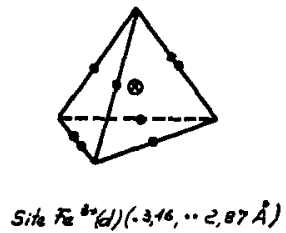

(c)

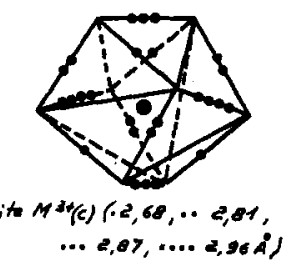

The distances are relotive to $5 \mathrm{~F}_{4}, \mathrm{O}_{3} .3 \gamma_{2} \mathrm{O}_{3}$

Fig. 2. Positions of the oxygen and magnetic ions on the $a, d$ and $c$ sites (from Geller and M. A. Gilleo ${ }^{20}$ ). 
the variation of magnetisation with field can be represented by the linear expression

$$
J=J_{\mathrm{s}}+\chi H,
$$

except near liquid helium temperatures; the same expression also describes the behaviour of thulium and ytterbium ferrites except at very low temperatures.

For each ferrite, Fig. 3 shows the temperature variation of the spontaneous magnetism expressed in Bohr magnetons $\left(\mu_{\mathrm{B}}\right)$ relative to one grammemolecule $5 \mathrm{Fe}_{2} \mathrm{O}_{3} \cdot 3 \mathrm{M}_{2} \mathrm{O}_{3}$. For the ferrites of gadolinium, terbium, dysprosium, holmium and erbium, the magnetisation is large at low temperatures, rapidly decreases as the temperature increases, becomes zero at a temperature $\theta_{\mathrm{c}}$ (Table 1), reappears, and then finally disappears at a Curie temperature $\theta_{\mathrm{f}}$ which is in the region of $560^{\circ} \mathrm{K}$ (Table 1) for these ferrites. These experimental results do not allow us to differentiate between a law in $T^{\frac{3}{3}}$ or $T^{2}$ for the approach to absolute zero; in either case one finds the magnetisation at absolute zero, $J_{0}$, by extrapolation (Table 1). For thulium ferrite, experiment allows us to determine the temperature $\theta_{\mathrm{c}}$ as being between 4.2 and $20.4^{\circ} \mathrm{K}$; for ytterbium ferrite the spontaneous magnetisation is small near absolute zero, $\theta_{c}$ being $7.7^{\circ} \mathrm{K}^{22}$; it then increases with the

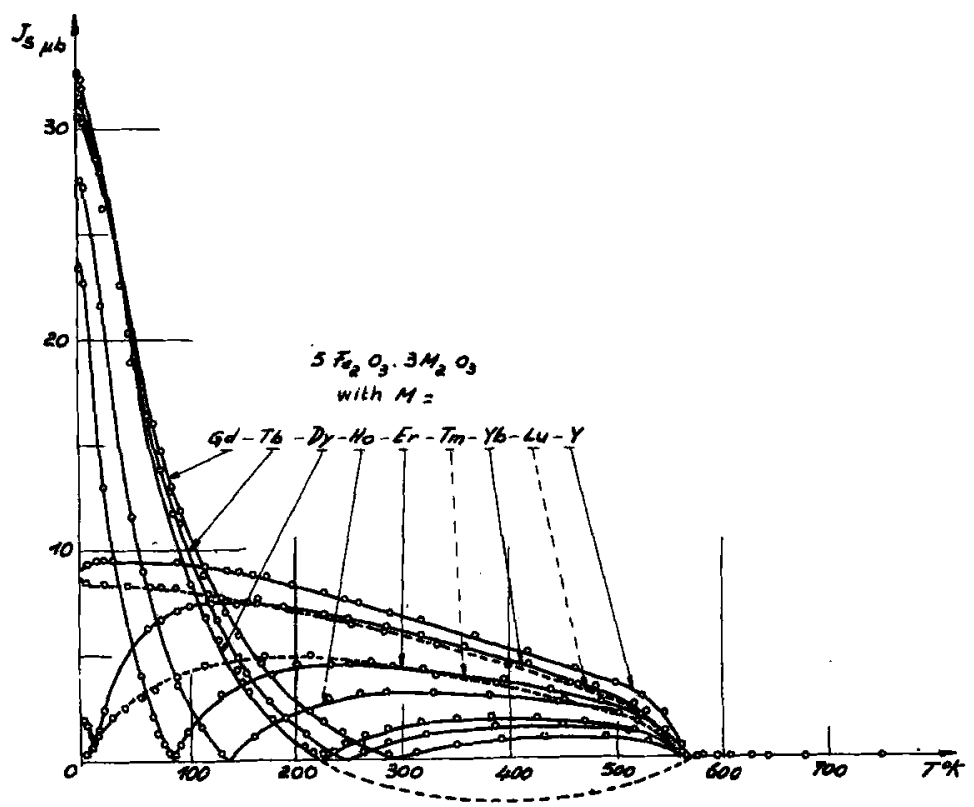

Fig. 3. Variations of the spontaneous magnetisation versus temperature for the magnetic garnets. 


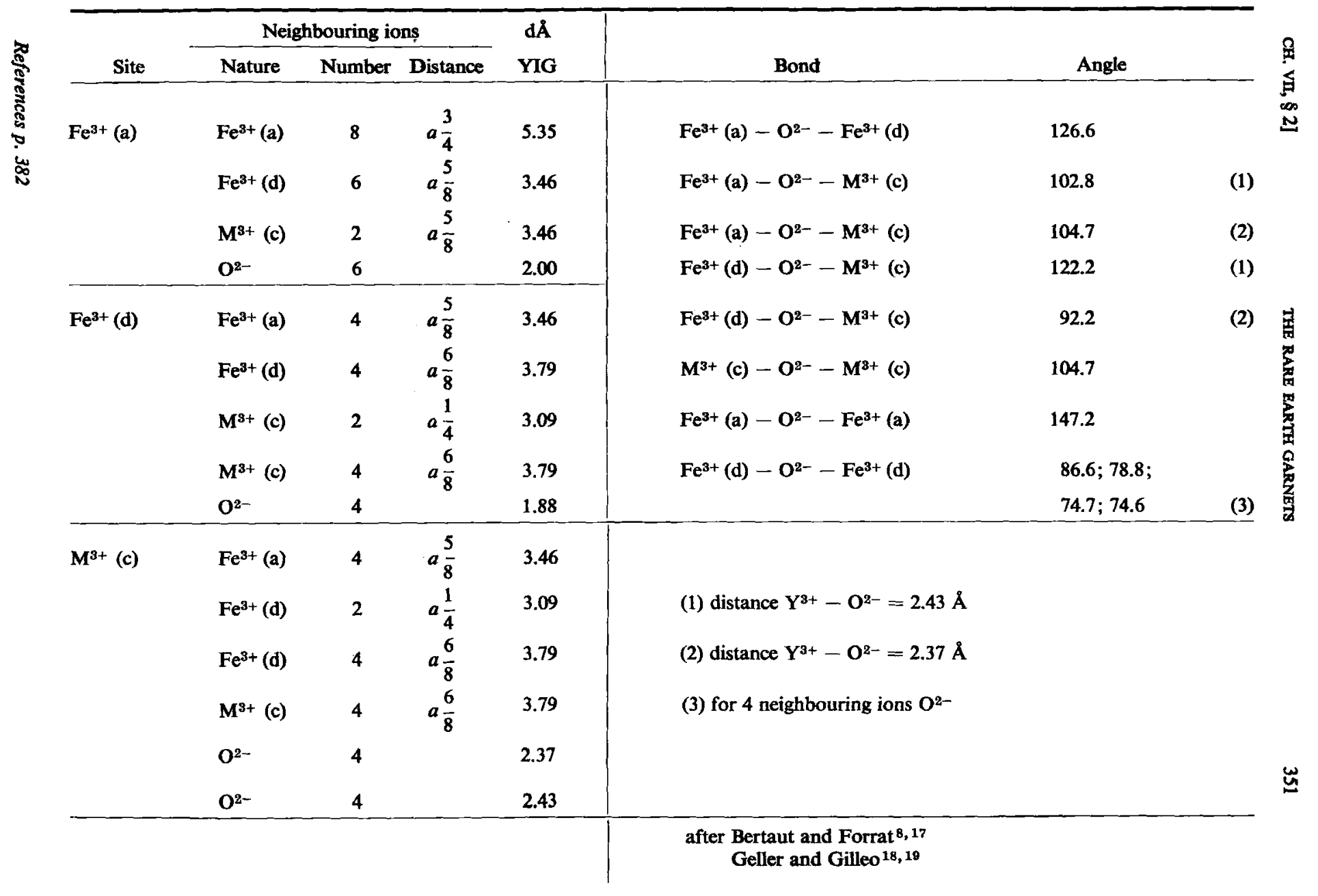


temperature, passes through a maximum decreases and becomes zero at $\theta_{\mathrm{f}}=548^{\circ} \mathrm{K}$. For the ferrites of yttrium, samarium, europium and lutetium, the variations $\left(J_{s}, T\right)$ are regular and similar to those of a normal ferromagnetic; the Curie points are about $560^{\circ} \mathrm{K}$.

Fig. 4 shows the variation with temperature of the inverse of the susceptibility, $\chi$, for one gramme-molecule of several compounds; also shown

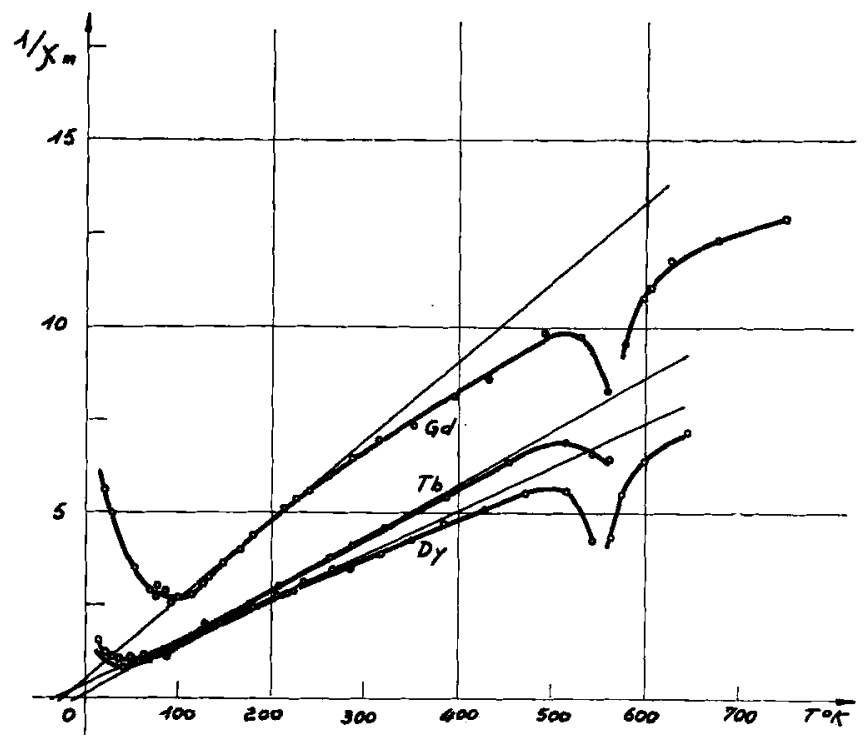

Fig. 4. Variations of the reciprocal superimposed susceptibility versus temperature, below Curie point, for some magnetic garnets.

are the Curie-Weiss lines defined by the theoretical value of the Curie constant $C_{\mathrm{c}}$ as deduced from the ground state of the free rare earth ion. Neglecting for the moment the region of saturation at very low temperatures and the region near the Curie point, we can see from the two curves that the susceptibility is very near that of the free rare earth ion. The order of magnitude of the paramagnetic Curie point, $\theta_{\mathrm{p}}$, is defined by extrapolation of the Curie-Weiss lines (Table 1); they are negative and near absolute zero; they give us, thus, an indication of the strength of magnetic interactions between the rare earth ions, namely that they are weak and negative.

These ferrites are paramagnetic above $570^{\circ} \mathrm{K}$ and their susceptibilities have been measured up to about $1500^{\circ} \mathrm{K}^{23}$. Note for the moment that the curves $\left(1 / \chi_{m}, T\right)$ (Fig. 5) are strongly concave towards the temperature axis, even more so than those observed for the spinel-type ferrites ${ }^{24,25}$; their 


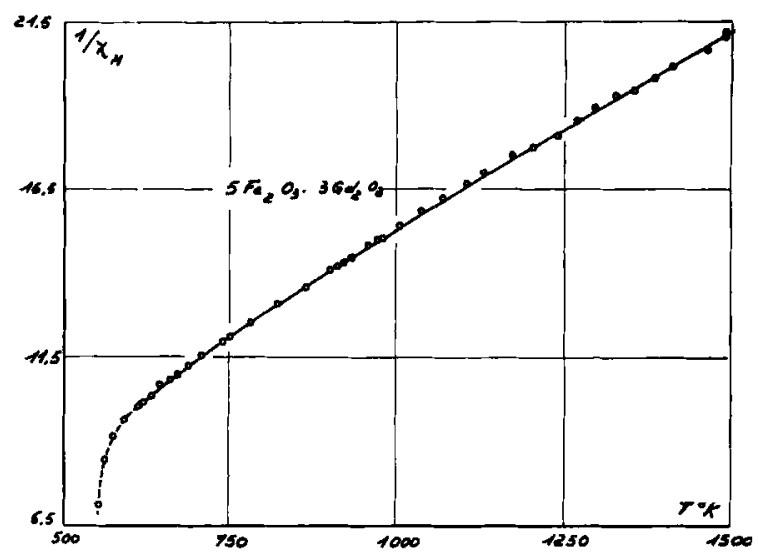

Fig. 5. Variation of the reciprocal susceptibility versus temperature, above Curie point, for Gd IG.

analysis yields important information on the magnetic interactions, which we shall treat in the following paragraph.

\subsection{MAGNETIC INTERACTIONS}

We shall firstly make some remarks particular to the magnetic behaviour of these ferrites.

Consider the ferrites of yttrium and of gadolinium; take the sum of the absolute values of the moments of the magnetic ions in the molecule at absolute zero. Knowing that the moment of a $\mathrm{Fe}^{3+}$ ion is $5 \mu_{\mathrm{B}}$, that of $\mathrm{Y}^{3+}$ is zero and that of $\mathrm{Gd}^{3+}$ is $7 \mu_{\mathrm{B}}$, we find the sums of $50 \mu_{\mathrm{B}}$ for $5 \mathrm{Fe}_{2} \mathrm{O}_{3} \cdot 3 \mathrm{Y}_{2} \mathrm{O}_{3}$ and $92 \mu_{\mathrm{B}}$ for $5 \mathrm{Fe}_{2} \mathrm{O}_{3} \cdot 3 \mathrm{Gd}_{2} \mathrm{O}_{3}$; that is, much higher than the respective measured values of $9.44 \mu_{\mathrm{B}}$ and $30.3 \mu_{\mathrm{B}}$.

Let us add that the temperature variation of the spontaneous magnetisation (Fig. 3) is quite different from that of iron, say; certain of these curves show a zero spontaneous magnetisation at a temperature below the Curie point which then reappears - just like that observed for the spinel-type ferrites of formula $\mathrm{Li}_{0.5} \mathrm{Fe}_{2.5-x} \mathrm{Cr}_{x} \mathrm{O}_{4}{ }^{26}$.

Also the variation of the inverse of the paramagnetic susceptibility with temperature above the Curie point is concave towards the temperature axis (Fig. 5).

These remarks show us a striking analogy between the observed behaviour of these ferrites and that observed for the spinel-type ferrites $25-27$; the interpretation of these results must thus be sought within the framework of a ferrimagnetic model 27. 
To account for the observed magnetisation at absolute zero we are led to adopt the following model: There are strong negative interactions between sub-lattices (a) and (d) which orient the $\mathrm{Fe}^{3+}$ ions on the octahedral sites in an antiparallel direction to the $\mathrm{Fe}^{3+}$ ions on the tetrahedral sites; the interaction between the ions on the sub-lattices (c) and (d) are negative but weak and the $\mathrm{M}^{3+}$ ions on sites (c) are magnetised in an antiparallel direction to that of the resultant moment of the $\mathrm{Fe}^{3+}$ ions. The values of the magnetisations at absolute zero for yttrium and gadolinium ferrites are, according to this scheme, $10 \mu_{\mathrm{B}}$ and $32 \mu_{\mathrm{B}}$ respectively; these are in good agreement with the observed values.

Let us now see if our model is compatible with the known facts on magnetic interactions in the ferrites. Being insulators, the magnetic interactions cannot occur via the conduction electrons in the garnet-type ferrites; the magnetic ions are separated from each other by the large oxygen ions and this separation is too large to give rise to an appreciable direct exchange 28 ; however this situation can give rise to important superexchange interactions ${ }^{29}$ in certain cases. The theory shows ${ }^{30}$ that these indirect exchange interactions decrease with the separation of the magnetic ions and increase as the angle formed by triplet $\mathrm{M}^{3+}-\mathrm{O}^{2-}-\mathrm{M}^{3+}$ tends to $180^{\circ}$. In the garnet structure the bond angle $\mathrm{Fe}^{3+}$ (a) $-\mathrm{O}^{2-}-\mathrm{Fe}^{3+}$ (d) is $126^{\circ} 6^{\prime}$ (Table 2) and the distance $\mathrm{Fe}^{3+}-\mathrm{O}^{2-}$ about $2 \AA$; this arrangement is very favorable for strong negative interactions between the sub-lattices a and $d$. Similarily the bond angle $\mathrm{M}^{3+}(\mathrm{c})-\mathrm{O}^{2-}-\mathrm{Fe}^{3+}$ (d) is $122^{\circ} 2^{\prime}$; the distance $\mathrm{M}^{3+}(\mathrm{c})-\mathrm{O}^{2-}$ is $2.43 \AA$, greater than the previous case; one can thus suppose that the $\mathrm{M}^{3+}(\mathrm{c})-\mathrm{Fe}^{3+}(\mathrm{d})$ interactions are negative and weaker than $\mathrm{Fe}^{3+}(\mathrm{a})-$ $\mathrm{Fe}^{3+}(\mathrm{d})$ interactions previously considered. The bond angles $\mathrm{Fe}^{3+}$ (a) $-\mathrm{O}^{2-}$ $-\mathrm{M}^{3+}(\mathrm{c})$ are $102^{\circ} 8^{\prime}$ and $104^{\circ} 7^{\prime}$, both near $90^{\circ}$; consequently these interactions are weak. Continuing this line of reasoning one can expect that the interactions between the iron ions on sub-lattice (a) are strong, the bond angle $\mathrm{Fe}^{3+}(\mathrm{a})-\mathrm{O}^{2-}-\mathrm{Fe}^{3+}$ (a) being $147^{\circ} 2^{\prime}$; on the other hand the interaction between the $\mathrm{Fe}^{3+}$ (d) ions should be weak as the bond angle lies between $74^{\circ} 6^{\prime}$ and $86^{\circ} 6^{\prime}$.

\subsection{INTERPRETATION OF EXPERIMENTAL RESULTS}

\section{A. Magnetisation at absolute zero}

Let us generalise this ferrimagnetic model for the rare earth ferrites, excepting samarium and europium. The $\mathrm{Fe}^{3+}$ ion is in an S-state with a well defined moment at absolute zero of $5 \mu_{\mathrm{B}}$; for the series of rare earth 
ions from terbium to ytterbium inclusive, the moment at absolute zero is $(L+2 S) \mu_{\mathrm{B}}$ for the free ion under the hypothesis of Russel-Saunders coupling; $L$ and $S$ are the orbital and spin quantum numbers respectively (Table 1). Thus the moment of $5 \mathrm{Fe}_{2} \mathrm{O}_{3} \cdot 3 \mathrm{M}_{2} \mathrm{O}_{3}$ at absolute zero must be

$$
J_{0}=[6(L+2 S)-10] \mu_{\mathrm{B}} \text {. }
$$

The agreement of the values calculated from the expression (Fig. 6) and those observed is satisfactory only for yttrium, gadolinium and lutetium ferrites; for the other ferrites the rare earth moment must be less than the theoretical value $(L+2 S) \mu_{\mathrm{B}}$. We already know that in the first series of transition

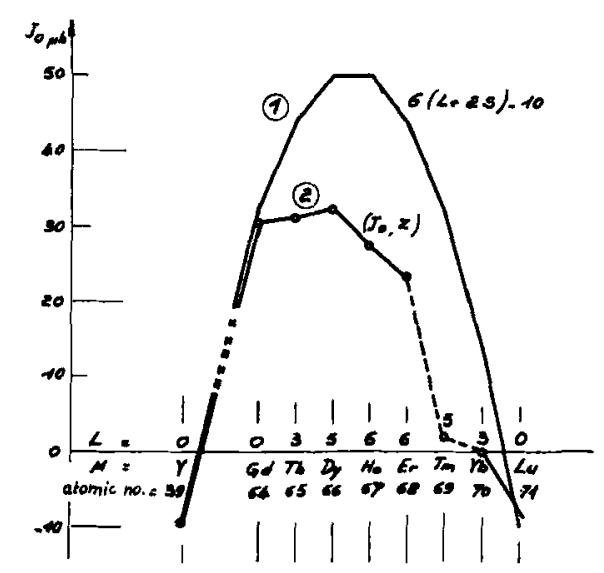

Fig. 6. The absolute saturation of the magnetic garnets.

elements the orbital moment is largely "quenched"; the magnetic moment is due mainly to the spin; also, that this is due to the asymmetry of the crystalline field acting on the magnetic ion. While this may not be exactly the same case for the rare earths in the garnet-type ferrites one can well conceive a similar "quenching". The rare earth ion is surrounded by eight oxygen ions at the vertices of an irregular hexahedron, which produce a strongly asymmetric crystalline field acting on the ion. This field raises the degeneracy of the energy levels; in particular, the moment of the ground level is no longer that of the free ion. We also note that the separations of these split levels from the ground level can be in the order of the energy corresponding to the measuring temperature; thus there is a contribution to the magnetisation from these sub-levels which is temperature dependent. The various theoretical investigations ${ }^{31-33}$ of this subject confirm our view- 
point. This ferrimagnetic model has also been confirmed by the neutron diffraction of yttrium ferrite at room temperature ${ }^{15,34}$.

\section{B. Yttrium ferrite. Magnetic interactions between iron ions}

We know that the Curie points of these ferrites lie in the narrow band 548 to $578^{\circ} \mathrm{K}$, whatever the rare earth ion (Table 1). From this we conclude that the Curie points are independent of the interactions between the rare earth ions, or, otherwise stated, these interactions are weak; it follows that the Curie points are determined by the interactions between the iron ions which are strong and of the same strength in the various ferrites. As the temperature increases, the spontaneous magnetisation of the rare earth ions decreases more rapidly than that of the iron ions, due the weak coupling between the former; the resultant spontaneous magnetisation, equal to the difference between these two contributions, has the sign of that of the $\mathbf{M}$ ions at low temperatures, is zero at the compensation temperature $\theta_{c}$ (where the magnetisations of the $\mathrm{M}^{3+}$ and $\mathrm{Fe}^{3+}$ ions are equal and opposite) and then changes sign above $\theta_{\mathrm{c}}$. We illustrate this behaviour, noting that the part of the curve $\left(J_{\mathrm{s}}, T\right)$, which is symmetric in the abcissae above $\theta_{\mathrm{c}}$, is the extension of the curve drawn below $\theta_{\mathrm{c}}$ (Fig. 3). The compensation temperature is found experimentally by following the thermal variation of the remanent magnetisation of a specimen in a strong field at low temperatures by means of a ballistic galvanometer; as the temperature rises the galvanometer deflection falls, is zero at $\theta_{c}$ and then changes sign for higher temperatures.

In an external field the variation of the magnetisation of the iron ions in this ferrite is very weak, which fact is also borne out by measurement on yttrium and lutetium ferrites. However, for temperatures above $50^{\circ} \mathrm{K}$, the magnetisation of the $M$ ions is far from saturation, and the resultant variation of the magnetisation of the specimen is that of the $M$ ions. This variation is superimposed on the magnetisation in zero external field, and is of a paramagnetic nature.

These thermal variations have been quantitatively interpreted by an extension of the molecular field approximation successfully developed by Néel ${ }^{27}$ to explain the magnetic properties of the spinel-type ferrites of two magnetic sub-lattices; the extension is to three magnetic sub-lattices in this case.

The relative proportions of magnetic ions on the sites $a, d$ and $c$ are respectively $\lambda=\frac{1}{4}, \mu=\frac{3}{8}, v=\frac{3}{8}$. The molecular field approximation reduces to the assumption that the action of the nearest neighbours on a $\mathrm{Fe}^{3+}$ (a) ion is equivalent to a magnetic field $\boldsymbol{h}_{\mathrm{a}}$ which is the sum of the three fields $\boldsymbol{h}_{\mathrm{aa}}, \boldsymbol{h}_{\mathrm{ad}}, \boldsymbol{h}_{\mathrm{ac}}$; the first $\boldsymbol{h}_{\mathrm{aa}}$, is due to the $\mathrm{Fe}^{3+}$ (a) ions; it is proportional to 
the magnetic moment of these ions, $J_{a}$, and to their number, $\lambda$; the field $\boldsymbol{h}_{\mathrm{ad}}$ represents the action of the neighbours $\mathrm{Fe}^{3+}(\mathrm{d})$; it is proportional to their magnetic moment $J_{\mathrm{d}}$ and their number $\mu ; \boldsymbol{h}_{\mathrm{ac}}$ and the molecular fields $\boldsymbol{h}_{\mathrm{d}}$ for the $\mathrm{Fe}^{3+}$ (d) ion and $\boldsymbol{h}_{\mathrm{c}}$ for the $\mathrm{M}^{3+}$ (c) ion are defined in a similar way. These three molecular fields are written

$$
\begin{aligned}
& h_{\mathrm{a}}=n_{\mathrm{aa}} \lambda J_{\mathrm{a}}+n_{\mathrm{ad}} \mu J_{\mathrm{d}}+n_{\mathrm{ac}} v J_{\mathrm{c}} \\
& h_{\mathrm{d}}=n_{\mathrm{da}} \lambda J_{\mathrm{a}}+n_{\mathrm{dd}} \mu J_{\mathrm{d}}+n_{\mathrm{dc}} v J_{\mathrm{c}} \\
& \boldsymbol{h}_{\mathrm{c}}=n_{\mathrm{ca}} \lambda J_{\mathrm{a}}+n_{\mathrm{cd}} \mu J_{\mathrm{d}}+n_{\mathrm{cc}} \nu J_{\mathrm{c}}
\end{aligned}
$$

where the molecular field coefficients, $n_{l j}$, are proportional to the exchange integrals.

At temperature $T$, in external field $(H)$, the partial magnetisations $J_{a}$, $J_{d}$ and $J_{c}$ are the solutions of the three simultaneous equations

$$
J_{i}=J_{0 i} B_{J i}\left\{\frac{J_{0 \mathrm{i}}}{R T}\left(h_{i}+H\right)\right\} ; \quad \mathrm{i}=\mathrm{a}, \mathrm{d}, \mathrm{c} .
$$

Here $J_{0 \mathrm{i}}$ is the moment at absolute zero of the ion on the site $\mathrm{i}$, and $B_{J_{\mathrm{i}}}$ is the Brillouin function of the ion i where $J_{i}$ is its quantum number. The spontaneous magnetisations $J_{\mathrm{as}}, J_{\mathrm{ds}}$ and $J_{\mathrm{cs}}$ are the solutions of the same equations with $H=0$; the resultant spontaneous magnetisation is

$$
J_{\mathrm{s}}=\lambda J_{\mathrm{as}}+\mu J_{\mathrm{ds}}+v J_{\mathrm{cs}} .
$$

At high temperatures the Brillouin function can be replaced by the first term of its series development and we have,

$$
J_{\mathbf{i}}=\frac{C_{\mathrm{i}}}{T}\left(\boldsymbol{h}_{\mathbf{i}}+\boldsymbol{H}\right)
$$

where $C_{\mathrm{i}}$ is the Curie constant of the ion $\mathrm{i}$.

When the rare earth ion is non-magnetic, as is the case with yttrium and lutetium ferrites, the problem reduces to that of a substance with two sublattices. We know that, theoretically, the thermal variation of the inverse of the paramagnetic susceptibility above the Curie point is a hyperbola ${ }^{24}$,

$$
\frac{1}{\chi_{\mathrm{m}}}=\frac{T}{C}+\frac{1}{\chi_{0}}-\frac{\sigma}{T-\theta},
$$

where $C$ is the Curie constant for all the magnetic ions in the ferrite and $1 / \chi_{0}, \sigma$ and $\theta$ are functions of the molecular field coefficients, $n_{\mathrm{ij}}$. 
2.6. Temperature Variation of the Inverse of the Paramagnetic Sus-

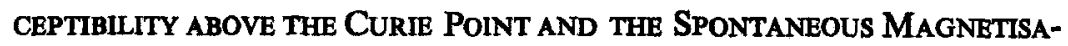
TION OF YTTRIUM FERRITE

We have applied the above treatment to the case of yttrium ferrite. From the experimental curve (Fig. 7) we have been able to fit a hyperbola of the above type which is a good approximation for the values $C=50,1 / \chi_{0}=$ $=30.5, \sigma=990$ and $\theta=570$. Before going further, it is necessary to note that the experimental Curie constant, $C$, is greater than the theoretical value

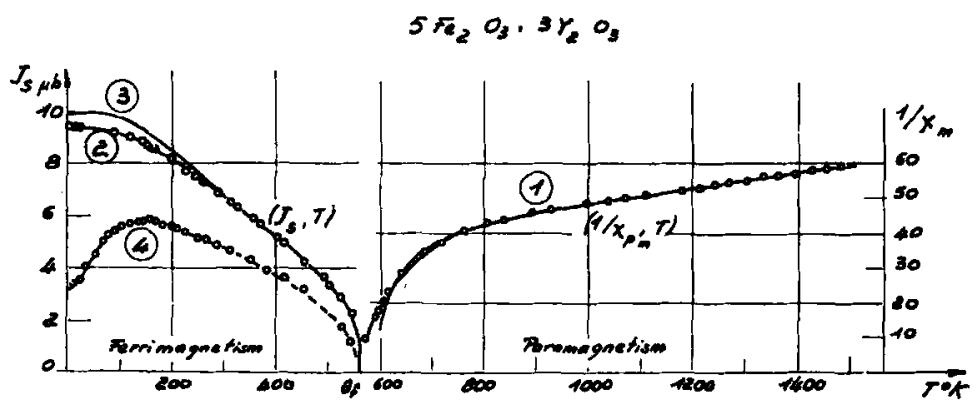

Fig. 7. Experimental and theoretical curves in the ferrimagnetic and paramagnetic range of temperature of YIG.

$C^{\prime}=43.77$ for the ten $\mathrm{Fe}^{3+}$ ions. The same result had already been found for the Curie constants of the spinel-type ferrites. Néel ${ }^{35}$ explained this as being due to the effect of thermal expansion on the magnetic interactions. On the hypothesis that the molecular field coefficient is a linear function of the temperature,

$$
n_{i j}=n_{0 i j}(1+\gamma T) \text {, }
$$

we have the following relation between $C$ and $C^{\prime}$ :

$$
\frac{1}{C}=\frac{1}{C^{\prime}}+\frac{\gamma}{\chi_{0}} \text {. }
$$

From this we deduce that $\gamma=-1.3 \times 10^{-4}$ for yttrium ferrite, which agrees well with the values observed for the spinel-type ferrites; knowing $1 / \chi_{0}$, $\gamma$ and $\theta$ this correction gives the following values of the molecular field coefficients: $n_{0 \mathrm{aa}}=-352, n_{0 \text { ad }}=-742$ and $n_{0 \mathrm{dd}}=-210$.

We note that these interactions are negative and that the strongest is between the magnetic ions of the sub-lattices(a) and (d), as expected. At 
very low temperatures the molecular field acting on a $\mathrm{Fe}^{3+}$ (a) ion is about $5.3 \times 10^{6}$ Oe and that on $\mathrm{Fe}^{3+}$ (d) ion $3 \times 10^{6} \mathrm{Oe}$.

Knowing the coefficients $n_{1 \mathrm{j}}$, we can now solve the set of equations (1) for a given temperature and $H=0$. We thus find the theoretical thermal variations of the spontaneous magnetisations of the iron ions on each sublattice, $\left(J_{\mathrm{as}}, T\right)$ and $\left(J_{\mathrm{ds}}, T\right)$; this also gives us the thermal variation of the total spontaneous magnetisation $\left(J_{\mathrm{s}}, T\right)$. Comparison with the experimental curve (Fig. 7.) shows satisfactory agreement with this theory.

Robert ${ }^{36}$ has recently obtained some very interesting experimental results for the thermal variation of $\left(J_{\mathrm{as}}, T\right)$ and $\left(J_{\mathrm{ds}}, T\right)$ in yttrium ferrite. Starting from Solomon's experimental results ${ }^{37}$ from the Mössbauer effect in this ferrite, he measured the nuclear magnetic resonance frequencies of the $\mathrm{Fe}^{57}$ ions on the a and $\mathrm{d}$ sites between 1.7 and $400^{\circ} \mathrm{K}$. Suppose that the hyperfine structure is given by a term $A S \cdot I, I$ being the nuclear spin and $A$ a constant independent of the temperature; because of the strong exchange coupling between the electron spins, each nucleus experiences an interaction dependent only on the mean value of the spin, $\langle S\rangle$, of the ion in the corresponding sub-lattice. The nuclear magnetic resonance frequency is thus $A\langle S\rangle$ and its variation measured against temperature is a sensitive measure of the mean value of the spin and hence of the spontaneous magnetisation at the site considered. Considering the approximations made, the agreement between these two types of curves, while not perfect, is satisfactory (Fig. 8).

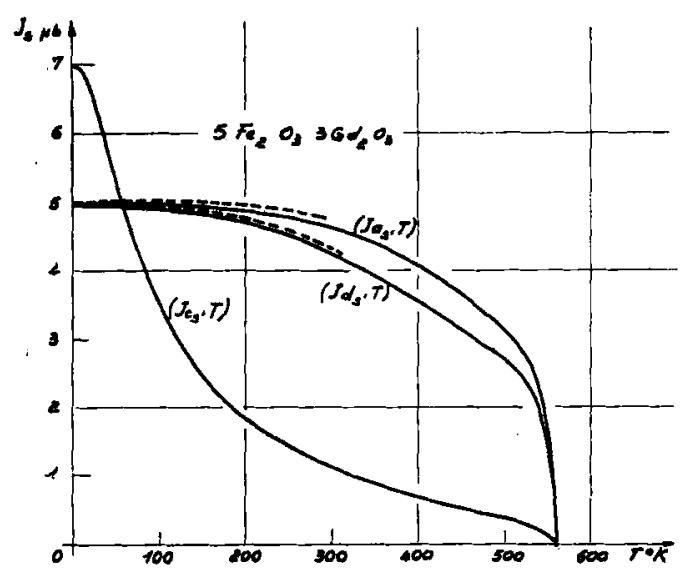

Fig. 8. Theoretical variations of the spontaneous magnetisations of the magnetic ions on the $a, d$ and $c$ sites in the case of $G d I G$. 
C. Garnet-type ferrites with magnetic rare earth ions. Magnetic interactions between iron and rare earth ions

Since the Curie points of these ferrites are of the same order of magnitude as that of yttrium ferrite, we shall suppose that the interaction between iron ions is the same in these ferrites as those we have just defined for the yttrium ferrite.

We shall take into account the magnetic interactions between iron and rare earth ions by defining a mean molecular field coefficient, $n$, such that its product by the sum of magnetisations of the iron ions is equal to resultant of the molecular fields $h_{\mathrm{ca}}$ and $h_{\mathrm{cd}}$, due to the ions $\mathrm{Fe}^{3+}$ (a) and $\mathrm{Fe}^{3+}$ (d) respectively; $\boldsymbol{n}$ thus satisfies the equation

$$
n\left(\lambda J_{\mathrm{as}}+\mu J_{\mathrm{ds}}\right)=n_{\mathrm{ca}} \lambda J_{\mathrm{as}}+n_{\mathrm{cd}} \mu J_{\mathrm{ds}} .
$$

At the compensation temperature equation 3 of the set (1) becomes

$$
J_{\mathrm{cs}}=J_{0 \mathrm{c}} B_{J_{\mathrm{c}}}\left\{\frac{J_{0 \mathrm{c}}}{R \theta_{\mathrm{c}}}\left(-n+n_{\mathrm{cc}}\right) v J_{\mathrm{cs}}\right\},
$$

from which, using the above results, we find

$$
n=-\frac{1}{v \chi_{c}} \quad \text { for } T=\theta_{c} .
$$

This value of $n$ is negative and can be directly deduced from experiment, as its absolute value represents the inverse of the paramagnetic susceptibility superimposed on the ferrimagnetism of the iron ions at the compensation temperature $\theta_{\mathrm{c}}$.

To relate $n$ to the exchange integral which it represents, we note that exchange coupling acts only by means of the spin angular momentum; we are thus led to represent the exchange integral by a coefficient $n^{\prime}$ related to $n$ by:

$$
n^{\prime}=n \frac{L+2 S}{2 S}
$$

the variations of $n^{\prime}$ from one rare earth to another should be due only to the variation in distance between the ions, all other parameters being constant (Table 3).

TA B L E 3

\begin{tabular}{c|cccccc}
\hline M & Gd & Tb & Dy & Ho & Er & Tm \\
\hline$n^{\prime}$ & -107 & -86 & -93 & -67 & -66 & -58
\end{tabular}


Wolf and Van Vleck ${ }^{38}$ have found $n^{\prime}=-103$ for europium ferrite by an entirely different method; this is in good agreement with the above values. However, with the exception of europium and gadolinium ferrites, we should not attribute more than an order of magnitude importance to these values; for the other ferrites, whose compensation temperatures decrease as the atomic number of the rare earth increases (Table 1) we must make corrections to take into account the saturation of the rare earth ion and the effect of the crystalline field on its moment. For example: the molecular field acting on a $\mathrm{Gd}^{3+}$ ion in gadolinium ferrite at $100^{\circ} \mathrm{K}$ and due to the iron ions is about $3.7 \times 10^{5} \mathrm{Oe}$; that is, about one tenth of the field acting on the ion irons.

\subsection{Magnetic Interactions between Rare Earth Ions}

We have already indicated that the experimental values of the paramagnetic Curie points, for the superimposed paramagnetic susceptibility below the Curie point, are not accurate; they are impossible to measure in the case of thulium and ytterbium ferrites; from the relation $\theta_{\mathrm{p}}=n_{\mathrm{cc}} \nu C_{\mathrm{c}}$, we find the molecular field coefficient, $n_{\mathrm{cc}}$, which characterises the interactions between the rare earth ions; consequently these coefficients are not well determined. Fortunately, their order of magnitude is small and we can neglect them in certain problems. Taking gadolinium ferrite at $100^{\circ} \mathrm{K}$ as an example, the rare earth interaction is equivalent to a field of about $50000 \mathrm{Oe}$; this is about one seventh of the field due to the iron ions acting on the rare earth ion, and about one hundreth of the interaction between iron ions.

\subsection{Thermal Variation of the Spontaneous Magnetization and of the INVERSE OF THE PARAMAGNETIC SUSCEPTIBILITY IN THE RARE EARTH FeR- RITES}

We suppose that the thermal variations of the spontaneous magnetisations $\left(J_{a s}, T\right)$ and $\left(J_{\mathrm{ds}}, T\right)$ of the iron ions on sites a and $\mathrm{d}$ are the same as those established in examining yttrium ferrite (Fig. 8). From the known values of $n$ and $n_{\text {ce }}$, we deduce the spontaneous magnetisation of the rare earth ion $\left(J_{\mathrm{cs}}, T\right)$, as the solution of the equation

$$
J_{\mathrm{cs}}=J_{0 \mathrm{c}} B_{J}\left\{\frac{J_{0 \mathrm{c}}}{R T}\left(n\left[\lambda J_{\mathrm{as}}+\mu J_{\mathrm{ds}}\right]+n_{\mathrm{cc}} v J_{\mathrm{cs}}\right)\right\},
$$

using our previous results. Because of the weak coupling between rare earth ions, the variation found as solution of this equation is quite different from that of the iron ions and is in agreement with our previous hypotheses. 
Knowing the thermal variations of the spontaneous magnetisation of each sub-lattice we can thus deduce the total resultant spontaneous magnetisation $\left(J_{s}, T\right)$. The inverse of the superimposed susceptibility is given by

$$
\frac{1}{v \chi_{\mathrm{c}}}=\left(T / v \frac{J_{0 \mathrm{c}}^{2}}{R} B_{J c}^{\prime}(z)\right)-n_{\mathrm{cc}},
$$

where $B_{J 0}^{\prime}(z)$ is the derivative of the Brillouin function with respect to $z$ for the ion $\mathbf{M}$ at the value corresponding to the spontaneous magnetisation, $J_{c s}$, and the relevant temperature.

The inverse of the paramagnetic susceptibility above the Curie point is theoretically a third degree equation,

$$
\frac{1}{\chi}=\frac{T}{C}+\frac{1}{\chi_{0}}-\frac{\sigma T+\alpha}{T^{2}-\theta T+\beta} .
$$

Generally, for a substance with $p$ sub-lattices, such a curve is of degree $p$. Aléonard ${ }^{23}$ has developed these calculations and has attempted to determine all the coefficients $n_{i j}$, particularly those for the rare ion; this work is interesting, but it does not bring to light any more fundamental information on the interactions than that already developed.

\subsection{The Rare Earth Gallates}

We have been able to affirm that the rare earth ion plays an important role in the magnetic properties of the garnet-type ferrites. The form of the thermal variation of the spontaneous magnetisation is determined by the rare earth ion and we have also seen that the moment of this ion at low temperatures is less than that of the free ion. In order to fix the magnetic behaviour of this ion it is interesting to study its properties in a garnet-type structure where the only magnetic ions are those of the rare earths themselves; this is realised by substituting a non-magnetic ion such as gallium, aluminium, indium, scandium or chromium for the ferric ion in the ferrite. This substitution is most complete for gallium, with which it has been possible to prepare gallates with yttrium and all the rare earths from praesodymium to lutetium except prometheum. The crystal parameters vary between 12.25 and $12.57 \AA$, which is of the same order of magnitude as in the ferrites; we may also assume that the distances between rare earth ions and thus the dimensions of the polyhedron of oxygen ions surrounding the rare earth ion, are the same in the ferrites and the gallates; thus, the exchange interactions between rare earth ions are very weak. The very accurate measurements by Wolf et al. ${ }^{38}$ giving $\theta_{\mathrm{p}}=-0.048 \pm 0.01^{\circ} \mathrm{K}$ for $5 \mathrm{Ga}_{2} \mathrm{O}_{3} \cdot 3 \mathrm{Yb}_{2} \mathrm{O}_{3}$ 
confirm our point of view. More particularily, we shall examine the magnetic behaviour of ytterbium gallate at low temperatures. Starting from the variation of $(J, H)$ measured at temperatures $2.35,4.2$ and $20.4^{\circ} \mathrm{K}$ we have plotted $J / J_{0 c}$ as a function of $H / T^{39}$, where $J_{0 c}$ is the moment of $\mathrm{Yb}^{3+}$ at absolute zero in its ground state $\frac{7}{2}$. The curve thus obtained is unique (Fig. 9) and differs considerably from that of the Brillouin function for $\frac{7}{2}$.

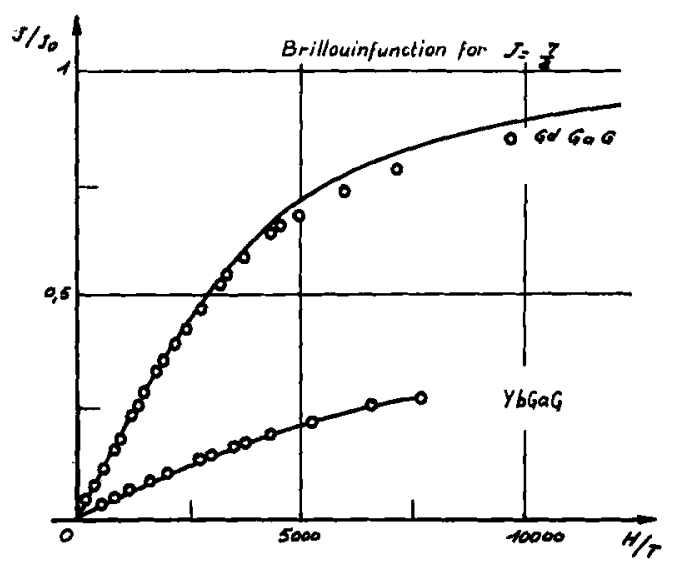

Fig. 9. Variations of the relative magnetisations versus $H / T$ for $\mathrm{Gd} \mathrm{Ga} \mathrm{G}$ and $\mathrm{Yb} \mathrm{Ga} \mathrm{G}$ and the corresponding Brillouin function.

On the other hand, the same curve plotted for gadolinium gallate, relative to the value of the moment of $\mathrm{Gd}^{3+}$ in an molecular field, shows that the moment of this ion is given by the Brillouin function even for large values of $H / T$. The difference in the magnetic behaviour of these two ions illustrates the "quenching" of magnetic moments in rare earth ions due to the crystalline field caused by the neighbouring oxygen ions. The ground state of $\mathrm{Gd}^{3+}$ is ${ }^{8} \mathrm{~S}$ and this is not split by the crystalline field; on the other hand the $\mathrm{Yb}^{3+}$ ion has ${ }^{2} \mathrm{~F}_{\frac{3}{2}}$ for its ground state; assuming a cubic crystalline field, this eight-fold degenerate level is split into two doublets, $\Gamma 6$ and $\Gamma 7$ and a quadruplet, $\Gamma 8$ (Fig. 10); under the real orthorhombic symmetry, the quadruplet is further split into two doublets. The ground level is $\Gamma 7$ and it is little altered by the orthorhombic symmetry; under these conditions of cubic symmetry the theoretical calculation of the magnetic properties of $\mathrm{Yb}^{3+}$ are more easily effected ${ }^{31}$. The magnetic moment is determined by the matrix elements for the states $I 7$ which give a term in $H / T$, and by 
the non diagonal elements between $\Gamma 7$ and $\Gamma 8$ which give a constant paramagnetic term. The latter may be obtained from the paramagnetic susceptibility 40 curve and one then can deduce the energy-gap between $\Gamma 7$ and $\Gamma 8$; this has the amazingly large value of $570 \mathrm{~cm}^{-1}$, which, however, has been confirmed independently by optical absorption measurements $\mathrm{s}^{41}$. It follows that the constant paramagnetism is small; it is negligible at low temperatures compared to the term in $H / T$ which can be exactly calculated, and

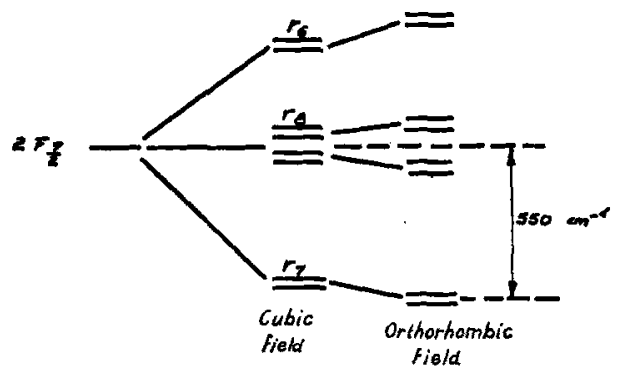

Fig. 10. The energy levels of the $\mathrm{Yb}^{3+}$ ion.

its theoretical variation is in good agreement with the experimental curve (Fig. 9). The departure from cubic symmetry at each site $c$ gives rise to an anisotropic Landé $g$-factor, which has been experimentally observed both by paramagnetic resonance ${ }^{42}$ and by anisotropy measurements in a static field ${ }^{43}$; it has been explained by a treatment of the actual orthorhombic symmetry ${ }^{40}$.

A second feature particular to the rare earth ion is its paramagnetic behaviour at high temperatures. As the exchange interactions are negligible, it is interesting to compare the product $\chi T$, of the paramagnetic susceptibility with the temperature, with the Curie constant $C$ of the ground level (Fig. 11). Agreement is perfect in the case of gadolinium gallate; for erbium and ytterbium $\chi T$ tends assymptotically to $C$ while for praesodymium, neodymium, samarium and europium gallates $\chi T$ increases continuously with the temperature; finally, for the gallates of terbium, dysprosium and holmium, $\chi T$ starts by increasing with the temperature, tends towards $C$ and then decreases for temperatures above about $900^{\circ} \mathrm{K}$.

These results typify a general type of paramagnetism in the rare earths that Van Vleck ${ }^{44}$ developed to interpret the susceptibilities of $\mathrm{Sm}^{3+}$ and $\mathrm{Eu}^{3+}$. For these latter at ambient temperatures the multiplet splitting is in the order of the thermal agitation energy, and these higher multiplet 
levels contribute progressively to the paramagnetism; for the other rare earths, the multiplet splitting is much larger, and the magnetic contribution due to the higher levels is negligible at ambient temperatures; at higher temperatures of about $1500^{\circ} \mathrm{K}$, where measurements have been made, this contribution becomes appreciable. Using Van Vleck's results for the paramagnetic susceptibility $\chi$, we have,

$$
\chi=\frac{N \sum_{J=(L-S)}^{J=(L+S)}\left[g_{J}^{2} \mu_{\mathbf{B}}^{2} \frac{J(J+1)}{3 K T}+\alpha_{J}\right](2 J+1) \mathrm{e}^{-W_{J} / K T}}{\sum_{J=L-S}(2 J+1) \mathrm{e}^{-W_{J} / K T}} .
$$

$W_{J}$ is the energy of the state with quantum number $J$ and $\alpha_{J}$ is the constant paramagnetism term; it is the sum of the squares of the non-diagonal matrix elements. The expression may be evaluated either from experimental values for the energy levels ${ }^{45}$ or by direct calculation of these levels ${ }^{44}$; it is then necessary to adjust the screening constant $\sigma$. Satisfactory agreement is obtained for $\sigma=34$, which is quite compatible with that used by Van Vleck in his study of $\mathrm{Sm}^{3+}$ and $\mathrm{Eu}^{3+}$.

\subsection{EURopium and Samartum Ferrites}

As we know the magnetic behaviour of the ions $\mathrm{Sm}^{3+}$ and $\mathrm{Eu}^{3+}$, we have
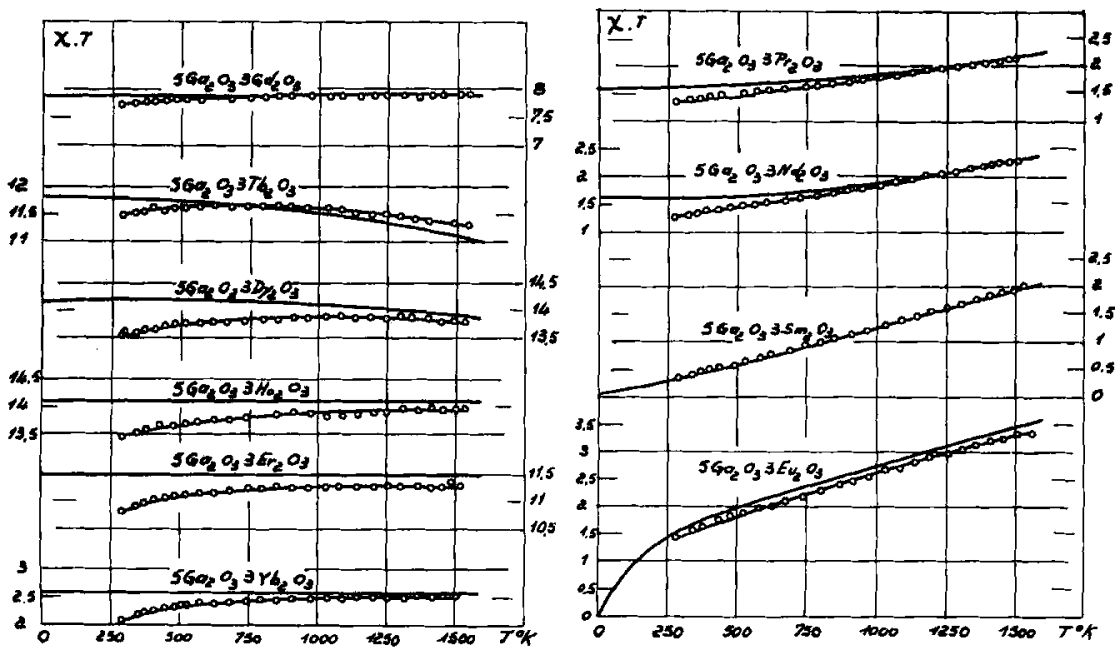

Fig. 11. The paramagnetism of the gallium garnets and the theoretical curves from Van Vleck's theory. 
left until now a discussion of their ferrites. We shall do so in light of what we have just seen for their gallates. The ferrimagnetic behaviour of these ferrites below their Curie points has been treated by Wolf and Van Vleck ${ }^{38}$ and by Van Vleck ${ }^{46}$; it may be summarised as follows. In the approximation of cubic symmetry the effect of the crystalline field on the ground state, ${ }^{7} \mathrm{~F}_{0}$, of $\mathrm{Eu}^{3+}$ may be ignored, as this is a state with $J=0$; also, the first excited level with $J=1$ is not split by a cubic field. The interactions between the rare earth ions may also be neglected as compared to the interactions between the iron ions which may be represented by a molecular field $H_{\mathrm{m}}=$ $n^{\prime}\left(\lambda J_{a s}+\mu J_{d s}\right)$. In zero external field, the rare earth ion is magnetised by the molecular field alone; the calculation of the resulting spontaneous magnetisation is of the type developed by Van Vleck ${ }^{44}$ to find the magnetisation of the free ion; one must consider both diagonal and non-diagonal terms for the different levels of the multiplet. Here, the molecular field plays the part of the external field but, one must remember in calculating the terms, that this molecular field acts only on the spins. Wolf and Van Vleck have done these calculations for the first three levels $J=0,1$ and 2; they adjusted $n^{\prime}$ such that there was agreement with the experimental value of $J_{s}$ at absolute zero; their value of $n^{\prime}=103$ is in agreement with ours of $n^{\prime}=107$ which was determined from gadolinium ferrite by quite different method. Knowing the thermal variation of the spontaneous magnetisation of the iron ions (taken as that of yttrium ferrite), they were able to calculate the thermal variation of the spontaneous magnetisation of both the $\mathrm{Eu}^{3+}$ ion and that of its corresponding ferrite. This latter variation is in perfect agreement with the experimental curve.

This same treatment has not been able to account for the magnetic properties of samarium ferrite in a quantitative manner ${ }^{46}$; it presents, however, the interest that at a certain temperature the moment of $\mathrm{Sm}^{3+}$ in the exchange field must change sign. There is an important contribution to the susceptibility from the non-diagonal elements between levels $\frac{5}{2}$ and $\frac{7}{2}$; this represents a constant moment. The diagonal terms vary as $1 / T$ and are of opposite sign to the non-diagonal ones; thus, the sum of the two changes sign at a certain temperature. This effect has not yet been studied in samarium ferrite but it has been observed in the alloy $\mathrm{Sm} \mathrm{Al}^{2}$ (ref. ${ }^{47}$ ). It could explain the low value of the moment of $\mathrm{Sm}^{3+}$.

\subsection{Suhstitutions in the Rare EARTh FerRites}

The problem of the substitution of the various ions (magnetic or not) in the rare earth compounds is interesting in that it gives us information on 
magnetic interactions, the properties of a particular ion and, like for the substitution by zinc in the spinel-type ferrites, it can have a practical interest. The substitution of the ferric ions was the first to be thought of ${ }^{48-50}$; it is complete with the ions $\mathrm{Al}^{3+}$ and $\mathrm{Ga}^{3+}$ and is partially complete with $\mathrm{Cr}^{3+}, \mathrm{Si}^{3+}$ and $\mathrm{In}^{3+}$. As the $\mathrm{Al}^{3+}$ and $\mathrm{Gd}^{3+}$ ions are smaller than the iron ones, they preferentially occupy the smallest crystallographic positions; that is the tetrahedral sites, $24 \mathrm{~d}$. The $\mathrm{Si}^{3+}$ and $\mathrm{In}^{3+}$ ions are bigger than those of iron, and they preferentially occupy the octahedral sites $16 a$. $\mathrm{A} \mathrm{Cr}^{3+}$ ion, which is much smaller than an iron one, preferentially occupies an octahedral site; this is probably as a result of its electronic configuration $\mathrm{d}^{2} \mathrm{sp}^{3}$ which is favorable to the formation of an octahedral bond. Next we may consider substitution of the rare earth ion. We have seen that the garnet-type ferrites exist for the rare earths between samarium and lutetium; evidently one can effect a substitution by any rare earth in this group. However the main interest is that of substitution by the lightest rare earths, $\mathrm{La}, \mathrm{Ce}, \mathrm{Pr}, \mathrm{Nd}$, which do not give simple ferrites as their ionic radii are too large. However one can have partial substitution in compounds of the type $5 \mathrm{Fe}_{2} \mathrm{O}_{3} \cdot(3-x) \mathrm{Y}_{2} \mathrm{O}_{3} \cdot x \mathrm{M}_{2} \mathrm{O}_{3}$ with $\mathrm{M}=\mathrm{La}^{3+}, \mathrm{Pr}^{3+}, \mathrm{Nd}^{3+} 51-53$; the limiting values of $x$ are 2 for $\mathrm{Nd}^{3+}, 1$ for $\mathrm{Pr}^{3+}$ and 0.5 for $\mathrm{La}^{3+}$. Cerium cannot be introduced into this structure. From what has been said on the ferrimagnetic model of the garnet-type ferrites, the substitution of a magnetic ion (e.g. neodymium) for $\mathrm{Y}^{3+}$ should decrease the resulting moment, because the rare earth moments are antiparallel to those of the iron ions; in fact experiment shows that the resultant moment of the ferrite increases with the amount of neodymium. This unexpected result has been explained by Wolf ${ }^{54}$. In the molecular field model we wrote the exchange energy as

$$
H_{\mathrm{ex}}=-n^{\prime} S_{\mathrm{Fe}} \cdot S_{\mathrm{TR}} \mu_{\mathrm{B}}^{2}
$$

where $S_{\mathrm{Fo}}$ is the resultant spins of the iron ions on the a and $\mathrm{d}$ sites and $S_{\mathrm{TR}}$ is the rare earth spin. As the coefficient $n^{\prime}$ is negative, these two spins are antiparallel in equilibrium. For the second rare earth sequence (gadolinium to ytterbium) the magnetic moment of the rare earth is parallel to the spin direction $(J=L+S)$; consequently the rare earth moment is in the opposite direction to that of the resultant moment of the iron ions on the $a$ and $d$ sites, and the moment of the ferrite is the difference between these two moments. For the first sequence (cerium to europium) the magnetic moment of the rare earth is in the opposite direction to its spin $(J=L-S)$ and hence the rare earth moment is to be added to the resultant moment of the iron ions. 


\subsection{OBservation of Elementary domains}

Slices of the rare earth ferrites (type garnet and gallate), of thickness about 20-50 microns, are transparent to visible light; they can form the basis of transmission experiments which are important from both a fundamental and applied viewpoint. Dillon ${ }^{55}$ was the first to observe wide transmission and absorption bands in the visible spectrum for yttrium ferrite. He observed a Faraday rotation of a plane polarised beam propagated parallel

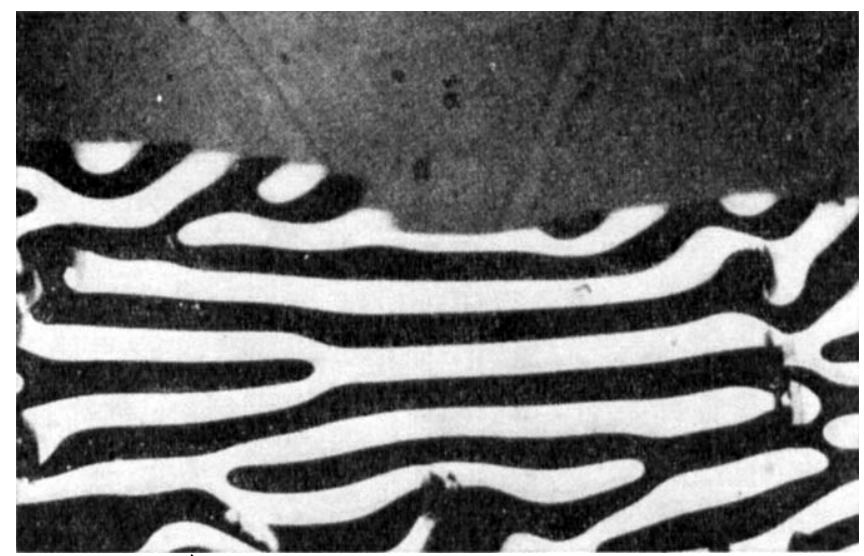

Fig. 12. Magnetic domains in YIG (from Dillon ${ }^{80}$ ).

to the direction of spontaneous magnetisation, the birefringence of a plane polarised beam propagated in a direction perpendicular to the spontaneous magnetisation and the dichroism for circularily polarised light.

An important practical application of this optical Faraday effect has been the direct observation of the elementary domains ${ }^{55-57}$. If a slice of a monocrystal is examined under a polarising microscope one sees an image generally ressembling Fig. 12 . The regions of different contrasts represent the domains magnetised in different directions; the maximum contrast is that for two domains at $180^{\circ}$ and whose magnetisations are parallel and antiparallel to the optic axis. By adjusting the analyser with respect to the polariser, one can vary the contrast; when polariser and analyser are parallel there is no contrast between the domains themselves, but the Bloch walls show up as black lines. One can also follow the movements of these Bloch walls in an external field and see their disappearance at the Curie point.

We point out that the observed domains are those of the whole of the 
sample, and not just those of its surface as is the case for the Bitter powder technique. However, the specimens must be very thin and the domain structure depends on the surface state - unless all contrast has disappeared. This is difficult to realise in practice as one can use neither electrolytic polishing nor chemical etching; but with careful mechanical polishing excellent patterns may be observed.

\section{The Levels of Rare Earth Ions in the Garnets (by B. DREYFus)}

As we have seen in the first part of this article, the central problem in the study of the garnets is the determination of the magnetic properties of the rare earth ions. They are acted on by a non-cubic crystal field and an exchange field due to the $\mathrm{Fe}^{+3}$ ions. Since the orientation of the crystal field, with respect to the cubic axes of the crystal, varies from one ion in the unit cell to the next (for a given general direction of the magnetisation there are six different sites), and further, since the splittings due to the crystalline and exchange fields are not always much larger than the thermal agitation energy, the resulting situation is extremely complicated and this has attracted the notice of many research workers. In the following paragraphs we shall examine some of these investigations devoted principally to the low temperature region and the determination of the rare earth levels; we refer the reader to the original articles where he will find a much more complete set of references. In particular, it has not seemed possible to consider the very many investigations devoted to paramagnetic and ferrimagnetic resonance, the Mössbauer effect, nuclear resonance, etc.

\subsection{Crystaline Fields}

We know that the internal coupling in the $4 \mathrm{f}$ shell leads to a term with a given $L$ and $S$ (following Hund's rule, generally). The spin-orbit coupling splits the term into corresponding multiplets labelled with the quantum number $J(J=L+S)$. The elements to the right of gadolinium in the periodic table have $J=L+S$, whilst those to the left have $J=L-S$; gadolinium itself has an orbital moment $L=0$. This description corresponds to the free ion, but the rare earth ion in a crystal is subject to the perturbation of the crystalline field which is due to its environment; this field partially raises the degeneracy of the ground multiplet. The crystalline field is characterised by its symmetry and its strength. These questions have been treated by Bethe in his classic paper of 1929 where he shows how the theory of groups can simplify the problem by taking fullest advantage of the symmetry of the crystal. 
In the garnets the determination of the energy and the properties of each sub-level (gyromagnetic ratio and splitting due to an external or exchange field) is indispensable for the understanding of the many macroscopic properties: susceptibility, spontaneous magnetisation, anisotropy, optical absorption spectra, etc.

The crystalline field acting on the $4 \mathrm{f}$ electrons can be developed in terms of spherical harmonics, $Y_{l}^{m}$, with $l=2,4$ or 6 . We need only consider even terms, and terms higher than sixth order are zero. Various degrees of approximation have been used; firstly, that of a field of cubic symmetry in which only spherical harmonics of fourth and sixth order appear. White and Andelin ${ }^{32}$ have treated a cubic potential of fourth order together with a magnetic field in direction $[1,0,0]$ or $[1,1,1]$. The results obtained from a computer (the order of the matrices, $2 J+1$, can be as high as 17) give the energies as a function of the ratio of the strength of the magnetic field to the strength of the crystalline field. One has also taken into account sixth order terms to find the energies and wave functions when considering a crystalline field of cubic symmetry 58,50 . More refined calculations have tried to take into account the actual; non-cubic, symmetry ${ }^{60}$. Ayant and Thomas ${ }^{61}$ have used a distorted cube as their model, as well as a more rigorous one ${ }^{40}$ where they have replaced the true position of the oxygen atoms by point charges (electrostatic model). Finally, Dillon and Walker ${ }^{62}$ have performed certain more complete calculations regarding the orientation of the field.

\subsection{SPECIFic Heats}

The thermal excitation of the various energy levels gives rise to a contribution to the specific heat. We should note however that the rare earth ions in the crystal are not isolated, but are magnetically coupled to the $\mathrm{Fe}^{3+}$ ions. We then have collective oscillations (spin-waves or magnons), and one can speak of the energies of individual ions only with care.

Many measurements have been made on powdered garnets ${ }^{63-65}$. The most complete are those of Harris and Meyer ${ }^{60}$ in the range $1.3^{\circ} \mathrm{K}$ to $20^{\circ} \mathrm{K}$.

The specific heat may be considered as the sum of three terms

$$
C=C_{\text {latt }}+C_{\text {magn }}+C_{\text {nuel }} \text {; }
$$

$C_{\text {latt }}$ is the lattice contribution which may be characterised by a Debye temperature, $\theta$. Assuming similar structures, we can suppose that the force constants are the same for all the garnets to a first approximation, and thus $\theta \propto M^{-\frac{1}{2}}$ where $M$ is the molecular mass. We probably do not make a 
large error in taking $\Theta$ as that of $\mathrm{Lu} \mathrm{I} \mathrm{G} \mathrm{which} \mathrm{has} \mathrm{been} \mathrm{very} \mathrm{accurately}$ determined as $458^{\circ} \mathrm{K}$. The upper limit is that of YIG at $\theta=572^{\circ} \mathrm{K}$. The nuclear contribution, $C_{\text {nucl }}$, is due to the hyperfine coupling with the electronic moments, and gives rise to a Schottky anomaly; only its "tail", varying as $T^{-2}$, is observable in the temperature range studied. The contribution of the $\mathrm{Fe}^{57}$ can be estimated from the Mössbauer effect or by nuclear resonance; it is too small to be measured. However the contribution from the rare earth nuclei may be appreciable:

$$
C_{\mathrm{nucl}}=\frac{R}{T^{2}} \frac{I(I+1)}{3}\left(\frac{\Delta}{k_{\mathrm{B}}}\right)^{2}
$$

Here $C_{\text {nucl }}$ is for one mole of active nuclei (correcting for isotopic abundance), $I$ is the nuclear spin and $\Delta$ the energy separation between successive nuclear levels in the hyperfine field; the contribution due to quadrupolar coupling of the nucleus with the gradient of the electric field has been neglected.

In the absence of measurements below $1.3^{\circ} \mathrm{K}$, it has been possible to determine $\Delta$ only for Tb I G $\left(\Delta=0.10 \mathrm{~cm}^{-1}\right)$ and Ho I G $\left(\Delta=0.181 \mathrm{~cm}^{-1}\right)$, for which this term is the most important. These values are compatible with those determined by electronic paramagnetic resonance in the other rare earth salts. $\Delta$ may be calculated if one knows the exact electronic wave function for the ground level. One must take into account the inequivalence of the different sites. In particular, $C_{\text {nucl }}$ of a monocrystal depends on the orientation of the spontaneous magnetisation with respect to the axes. It seems that such a calculation has not been performed.

Finally, the last term, $C_{\text {mag }}$, is the most interesting. In YIG and Lu I G only the $\mathrm{Fe}^{3+}$ ions are magnetic, the anisotropy is weak, and the theory of spin waves gives a specific heat varying as $T^{\frac{3}{3}}$.

$$
C_{\text {mag }}=0.235\left(k_{\mathrm{B}} \frac{T}{D}\right)^{\frac{3}{2}} \mathrm{~J} / \mathrm{M} d^{\circ},
$$

where $D$ is the coefficient for the acoustic branch of the spin-waves of frequency $\omega$ and wave-vector $k$

$$
\hbar \omega=D k^{2} a^{2}
$$

( $a$ is the lattice parameter). $D$ can be calculated from the exchange integrals and is proportional to the sum

$$
\left[50 J_{\mathrm{aa}}-\frac{125}{4} J_{\mathrm{ad}}+\frac{75}{4} J_{\mathrm{dd}}\right] \text {. }
$$

Douglass ${ }^{67}$ has determined the spin-wave spectrum of YIG at the centre 
and corners of the Brillouin zone. His calculations show that it is only the acoustic branch of the spin-waves that is excited in an appreciable manner at $20^{\circ} \mathrm{K}$. Although the variation in $T^{\frac{3}{2}}$ is verified experimentally, the values of $D$ measured on several samples of YIG vary from 16 to $27 \mathrm{~cm}^{-1}$. For Lu I G the only value is $27.1 \mathrm{~cm}^{-1}$. These values do not agree perfectly with the exchange integrals calculated from magnetic measurements or by microwave instability ${ }^{68-69}$.

In the case of $\mathrm{Gd} \mathrm{I} \mathrm{G} \mathrm{the} \mathrm{spin-wave} \mathrm{frequencies} \mathrm{have} \mathrm{been} \mathrm{calculated}$ for the centre of the zone ${ }^{70}$. This shows that a certain number of optical branches have frequencies low enough to be excited at $20^{\circ} \mathrm{K}$ (there are 11 modes with frequencies between $27^{\circ} \mathrm{K}$ and $46^{\circ} \mathrm{K}$ ). Tinkham ${ }^{71}$ has shown that, due to the slight differences between the gyromagnetic ratios of the ions on different sites, the frequencies of the optical branch are practically constant for most of the zone; these collective excitations have a groupvelocity which, if not zero is very small, and are well represented by individual excitations having the same energy as the separations of the sublevels of the rare earth ions. This enables us to justify the simple model adopted by Harris and Meyer; they consider the sub-lattices (a) (d) and (c) as constituting a continuum in which spin-wave theory is valid; this gives a term in $T^{3}$; they then add the contribution due to the rare earth ions, treated as systems having individual energies, and under the influence of the molecular field due to (a) and (d). The separation of adjacent levels taken from their results is $\Delta E=28.6 \mathrm{~cm}^{-1}$, in good agreement with Pauthenet's magnetic measurements $\left(\Delta E=24.9 \mathrm{~cm}^{-1}\right)$. Detailed calculations of the magnon spectrum for the whole of the zone are now under way ${ }^{72}$.

The orbital moment is non-zero in the other garnets and we must accurately consider the action of the crystalline field. The example of $\mathrm{Yb}$ I G is the simplest; we must distinguish:

a. The contribution from the acoustic branch, varying as $T^{\frac{3}{2}}$. This is now smaller (than that of Y I G) due to the large anisotropy which converts this term into an exponential. We should also add that $D$ itself is increased because of the smaller degeneracy of the ground levels. The resultant specific heat is negligible.

b. The optical modes of the individual ions in the molecular field. These separate into two groups due to the inequivalence of the sites; when the spontaneous magnetisation is in the [111] direction the mean value of the corresponding splitting is $25 \mathrm{~cm}^{-1}$, which is in excellent agreement with the optical measurements of Tinkham and Sievers ${ }^{73,74}$ who find two rays at $23.4 \mathrm{~cm}^{-1}$ and $26.4 \mathrm{~cm}^{-1}$. 
c. A mode corresponding to the exchange resonance between the $\mathrm{Yb}^{3+}$ and $\mathrm{Fe}^{3+}$ ions which is accidentally situated at low frequencies. Calorimetric measurements give this mode a mean energy of $E=17 \mathrm{~cm}^{-1}$ while, for $k=0$, optical measurements give $E=14 \mathrm{~cm}^{-1}$.

We shall not discuss the other garnets (Ho, Tb, Er, Dy, Sm). Their general behaviour is well understood, but there are still many details to be explained.

\subsection{Thermal CONDUCtion}

We cannot leave the subject of spin-waves without drawing attention to the fact that their contribution to thermal conduction has recently 75,76 been shown. The measurements were on Y I G between $0.4^{\circ} \mathrm{K}$ and $20^{\circ} \mathrm{K}$. This substance was chosen because of its weak anisotropy and, hence, large magnon specific heat. The conductivity is given by the classical formula

$$
K=\frac{1}{3} \sum \operatorname{Cv\lambda }
$$

$v$ is the group velocity $\mathrm{d} \omega / \mathrm{d} k$, which is proportional to $k$ for the dispersion relation $\hbar \omega=D k^{2}$ and $\lambda$ is the mean free path.

Douglass has taken advantage of the influence of an external field, since the dispersion relation, $\hbar \omega=g \beta H+D k^{2}$, leads to a reduction of the specific heat, $C$; he thus separates the magnon and phonon contributions. He used a field of about $20 \mathrm{kOe}$ thus causing a gap of about $2^{\circ} \mathrm{K}$ in the spectrum. Apart from some complications due to the existence of domains, the magnon conductivity in zero field at $0.5^{\circ} \mathrm{K}$ has been estimated as $70 \%$ of the total conductivity. The magnon conduction follows a law in $T^{2}$, which agrees with the theory 77,78 for a free path independent of $k$. This free path is of the order of $2 \times 10^{-2} \mathrm{~cm}$ and is probably determined by paramagneticimpurity scattering which is very effective for long wave lengths.

\subsection{Spectroscopic Investigations}

The interaction of electromagnetic radiation with a large number of garnets has been studied both for powders and monocrystals, and principally by absorption. The lattice vibrations in the garnets make them strongly absorbent in a spectral region from about $100 \mathrm{~cm}^{-1}(100 \mu)$ to about $800 \mathrm{~cm}^{-1}$ $(12,5 \mu)^{79}$. We note in particular that the vibrations of the $\mathrm{SiO}_{4}$ tetrahedron can be detected; these are formed by the presence of $\mathrm{Si}^{4+}$ ions as impurities and play an important role in many properties (particularily anisotropy), due to the $\mathrm{Fe}^{2+}$ ions which appear to keep the crystal electrically neutral. We recall also the work on the optical absorption of the $\mathrm{Fe}^{3+}$ ions, whose 
spectrum starts at about $10000 \mathrm{~cm}^{-1}$ and has been studied up to about $35000 \mathrm{~cm}^{-180,81}$. Two transparent regions are left; the first is situated in the near infra-red and visible region where transitions between the ground and excited multiplets may be observed; the second is in the very far infrared (between $10 \mathrm{~cm}^{-1}(1000 \mu)$ and $\left.100 \mathrm{~cm}^{-1}(100 \mu)\right)$ where the principal transitions are those within the ground multiplet. We recall that in absorption measurements the line intensity is proportional to the population of the lower level. At very low temperatures the only transitions are those from the lowest sub-level; but at temperatures above that of liquid hydrogen $\left(k T \sim 15 \mathrm{~cm}^{-1}\right)$ we have the addition of a spectrum due to transitions from one of the excited sub-levels of the ground multiplet.

\subsection{The Visible ANd NeAR INFra-Red Spectrum}

Due to the inequivalence of the sites there are in general at least two spectra present, and more than two if the spontaneous magnetisation is arbitrarily inclined to the crystal axes. It seemed that one could get over the further complication of the exchange field splitting by using isomorphous samples such as gallates, aluminates, etc. but it appears that the crystalline field itself is quite sensitive to these substitutions, and that one should regard electrostatic models with prudence. The simplest case is that of $\mathrm{Yb}$ I G, studied by Wickersheim and White ${ }^{82,83}$ in the near infra-red.

$\mathrm{Yb}^{3+}$ has two multiplets, $J=\frac{7}{2}$ and $J=\frac{5}{2}$ (Fig. 13). The observed tran-

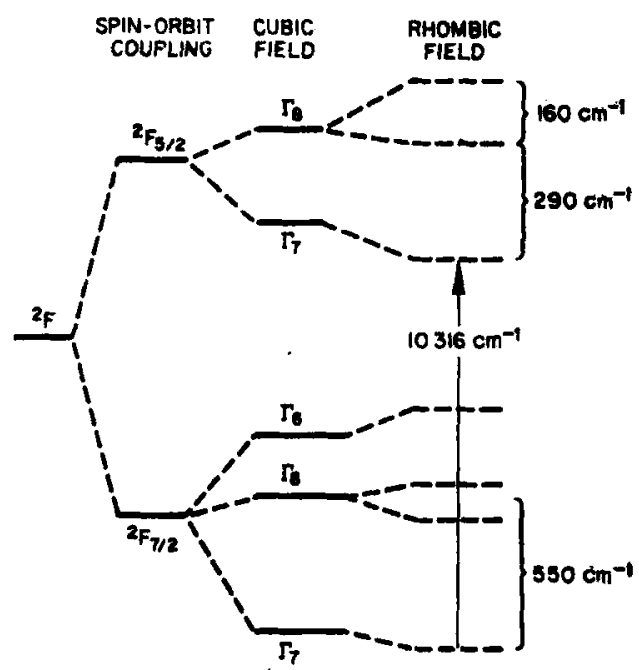

Fig. 13. Energy level scheme for $\mathrm{Yb}^{+++}$in $\mathrm{YGaG}$. 
sitions are from the ground sub-level to the various excited levels of the $J=\frac{5}{2}$ multiplet. The molecular field (whose effect is not shown in Fig. 13) splits each sub-level into two, and this splitting is different for the different sites. So that, at not too low temperatures $\left(T=77^{\circ} \mathrm{K}\right)$, there are four absorption lines centred on the $10316 \mathrm{~cm}^{-1}$ transition. In zero external field, the spontaneous magnetisation is along [111], there are two types of sites and hence eight lines. A study of the shift of these lines as a function of the angular variation of $M_{\mathrm{s}}$ allows one to deduce the Zeeman splitting. For example: the ground level for $M_{\mathrm{s}}$ parallel to [111] is split into two levels with separations $22.1 \mathrm{~cm}^{-1}$ and $25.3 \mathrm{~cm}^{-1}$ for the two sites (in good agreement with specific heat measurements and optical measurements in the far infra-red). The splitting as a function of angle can be written, using an effective field, as

$$
\mathscr{H}=-\boldsymbol{\mu} \cdot \boldsymbol{H}_{\text {eff }}
$$

where $\boldsymbol{\mu}$ is the magnetic moment due to the spin (as we are dealing with an exchange field) of the level considered.

$$
\boldsymbol{\mu}=\beta \underline{g}^{\prime} \mathbf{S}^{\prime},
$$

$S^{\prime}$ being an effective spin of $\frac{1}{2}$ and $\underline{g}^{\prime}$ the gyromagnetic tensor which can be found by electronic resonance (here the determination was on $\mathrm{Yb} \mathrm{Ga} \mathrm{G}$ ) ${ }^{42,84}$. The effective field is produced by the exchange interaction with the $\mathrm{Fe}^{3+}$ ions,

$$
\boldsymbol{H}_{\mathrm{eff}}=-\underline{\lambda} \cdot \boldsymbol{M}_{\mathrm{Fe}} .
$$

Thus, even when the exchange interaction is isotropic $(\lambda=$ constant $)$, it is natural that the splitting depends on the angle, because of the anisotropic $g$-factor. Now, optical measurements show that the exchange field itself is anisotropic, $\underline{\underline{\lambda}}$ being a tensor with the same principal axes as $g$. By way of example we give below the values of $H_{\text {eff }}$ and $g$ (for $\mathrm{Yb} \mathrm{GaG}$ ) in the three principal local directions of the rare earth ion.

TA B L E 4

\begin{tabular}{lccc}
\hline & $x$ & $y$ & $z$ \\
$H_{\text {ef: }}$ (gauss) & 87.200 & 153.000 & 169.000 \\
$g$ & 2.85 & 3.60 & 3.78 \\
$H_{\text {exeh }}=\frac{g J}{2(g J-1)}$ & 349.000 & 611.000 & 678.000 \\
$g J=8 / 7$ for $\mathrm{Yb}^{+++}\left({ }^{2} \mathrm{~F}_{7 / 2}\right)$ & & &
\end{tabular}


Starting from a detailed knowledge of the energy levels one can calculate macroscopic properties such as the spontaneous magnetisation of a monocrystal as a function of angle, its anisotropy etc. Such calculations have been performed ${ }^{22}$ and show good agreement with experiment.

Measurements have also been made on other garnets. The same $\mathrm{Yb}^{3+}$ ion has been studied diluted in $\mathrm{YGaG}^{60}$, which has the advantage of avoiding the complications caused by the molecular field; the splittings of the $\frac{7}{2}$ and $\frac{5}{2}$ multiplets were found and these agreed with susceptibility measurements ${ }^{31}$ which give about $500 \mathrm{~cm}^{-1}$ for the separation of the first excited level of the ground multiplet. The spectrum contains additional lines whose intensity is temperature dependent, thus bringing the lattice vibrations into evidence. In the case of $\mathrm{Er} \mathrm{I} \mathrm{G}^{85}$ the splittings and transitions of the ground multiplet $J=\frac{15}{2}$ and the excited multiplets $\frac{13}{2}$ and $\frac{9}{2}$, have been measured; the splitting of the ground level, as deduced from the hypothesis of an electrostatic cubic field, is in satisfactory agreement with the susceptibility of $\mathrm{Er} \mathrm{Ga} \mathrm{G}^{86}$. However, this simplified model does not seem able to completely explain the complexity of the spectrum.

\subsection{The far INFRA-Red Spectrum}

From $100 \mu$ the garnets become transparent again and it is possible to study transitions within the ground multiplet. This has been done by Tinkham and Sievers 73,74 between $10 \mathrm{~cm}^{-1}$ and $100 \mathrm{~cm}^{-1}$. The $10 \mathrm{~cm}^{-1}$ limit is not very important, since, if there were levels with lower energies, they would influence the specific heat. The spectra show several types of lines, amongst which one, at about $80 \mathrm{~cm}^{-1}$ and common to all the measured garnets, varies slowly as a function of molecular mass and has been attributed to lattice vibrations.

One next observes absorptions corresponding to the energies of the individual ions. This result, at first sight natural isn't completely so, since the electromagnetic waves have a large wavelength $(k \cong 0)$ and interact with the crystal as a whole. In reality, then, it seems that these absorptions are concerned with collective oscillations (spin-waves), particularily their optical branches. However, if all the sites (c) are identical, the optical modes for $k=0$ are magnetically compensated ${ }^{70}$ and no coupling with an electromagnetic wave is possible. Tinkham ${ }^{71}$ has shown that if the gyromagnetic ratios of the (c) ions are not quite the same, then the above selection rule is modified and absorptions corresponding to the optical branch become observable.

In the case where the $\mathrm{Fe}^{3+}$ ions are situated on the same sub-lattice 1 
and the identical rare earth ions on sub-lattice 2, there are two possible modes of resonance

$$
\omega_{0}=\frac{M_{1}-M_{2}}{M_{1} / \gamma_{1}-M_{2} / \gamma_{2}} H, \quad \omega_{\mathrm{e}}=-\lambda\left[\gamma_{2} M_{1}-\gamma_{1} M_{2}\right] .
$$

These modes are for $k=0$ in an external field, $H$, and neglecting anisotropy. $\lambda$ is the molecular field coefficient between 1 and 2 . The first mode is that of normal ferromagnetic resonance and is situated in the micro-wave region, except when we have to take into account a large anisotropic energy. The second is called exchange resonance ${ }^{87}$ and is normally found in the infrared; its intensity is proportional to $\left(\gamma_{1}-\gamma_{2}\right)^{2}$. One thus has two modes, neither of which correspond to individual transitions. The spectrum as a function of $k$, is shown in the upper half of Fig. 14. In the case of two slightly different sites (2), Tinkham has shown that the optical branch spectrum may be represented by considering half the preceding Brillouin-zone, that is, by folding back the dispersion curve (lower half of Fig. 14). For $k=0$ one now sees another mode with $\omega_{2}=\gamma \lambda M_{1}$ appear; this is in addition to the exchange-mode, and corresponds to the individual precession of ions (2) in the exchange field created by the ions (1). This mode has an intensity proportional to $\left(\delta \gamma_{2}\right)^{2}$ where $\delta \gamma_{2}$ is the difference between the

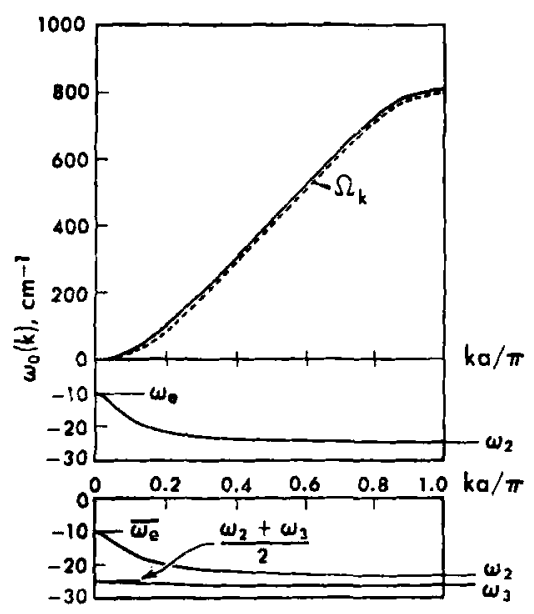

Fig. 14. Spin wave spectra of two and three sublattice models of rare earth iron garnets. Since the branch near the unperturbed iron spin-wave branch $\Omega_{k}$ is essentially the same in both cases, it is drawn only once. The two sublattice-case, shown above, gives one optical branch, and the three sublattice case gives two, as shown in the lower part of the figure. Note the tenfold difference in scale between the positive and negative frequency branches. 
gyromagnetic ratios of the two different (2) sites. We note in passing that for $k=0$ the dispersion of the branch $\omega_{2}$ is weak, which fact has been used to justify the Harris and Meyer model ${ }^{66}$ for the specific heat (see above). In as much as $\lambda M_{1}$ varies little between $0^{\circ} \mathrm{K}$ and $70^{\circ} \mathrm{K}$, the individual transitions are relatively insensitive to temperature variations; this has allowed their identification in the spectra.

In the case of Gd I G no transition has been observed, apart from that due to the lattice at $80 \mathrm{~cm}^{-1}$; this is explained by the fact that the $\mathrm{Gd}^{3+}$ ions are isotropic and, after what we have just said, the intensities of the individual transitions are thus zero. From magnetic and specific heat measurements, the exchange resonance ought to be at $28 \mathrm{~cm}^{-1}$. Now it accidentally happens that the gyromagnetic ratios of the $\mathrm{Fe}^{3+}$ and $\mathrm{Gd}^{3+}$ ions are both equal to 2 . The intensity of the corresponding transition is then zero; indeed, such a transition has not been observed.

$\mathrm{Yb}$ I G, already extensively studied in the visible and near infra-red, has been the object of an intensive investigation. One again finds the individual transitions ${ }^{80,83}$ at $23.1 \mathrm{~cm}^{-1}$ and $26.7 \mathrm{~cm}^{-1}$ for the two types of sites, and the magnetisation is parallel to [111]. The anisotropic gyromagnetic ratios and the exchange field have been found by application of an external field, variable in strength and direction. Although less precise than those of Wickersheim, these measurements agree with his and confirm the existence of an anisotropic exchange field.

Other, more complex, spectra have been observed (Sm I G, Ho I G, Er I G); in the case of $\operatorname{ErIG}$ and Sm I G it seems that the temperature variations can be explained only by considering the usually neglected interactions between rare earth ions.

Finally, the rest of the garnets shows transitions which are temperature dependent (for $\mathrm{Yb} \mathrm{I} \mathrm{G}$, they vary from $14 \mathrm{~cm}^{-1}$ at $0^{\circ} \mathrm{K}$ to $20 \mathrm{~cm}^{-1}$ at $70^{\circ} \mathrm{K}$ ); these transitions are attributed to the exchange resonance, $\omega_{\mathrm{e}}=$ $=-\left[\gamma_{2} M_{1}-\gamma_{1} M_{2}\right]$ which is temperature dependent, through the rare earth magnetisation, $M_{2}$.

In $\mathrm{Sm} \mathrm{I} \mathrm{G}$ a second temperature dependent transition has been identified as a ferromagnetic resonance; this latter is in the far infra-red due to a strong magnetocrystalline anisotropy, which acts as a strong temperature dependent effective field.

\subsection{InELASTIC SCattering OF NeUtrons}

The determination of the spin-wave spectrum, $\omega(k)$, is possible by the inelastic scattering of neutrons. The analysis is made from the diffracted 
neutrons, taking account of the conservation of energy and momentum. The dispersion of the optical branches is weak and the energy is practically that of the rare earth ion in the molecular field ${ }^{71}$. The energies of the $\mathrm{Yb}^{3+}$ ion have been found 88 at $80^{\circ} \mathrm{K}$ and $300^{\circ} \mathrm{K}$ in the case of $\mathrm{Yb} \mathrm{I} \mathrm{G.}$ The energies transferred are respectively $3.0 \mathrm{meV}$ and $2.4 \mathrm{meV}$, in agreement with magnetic, optical and thermal measurements. An energy transfer of $0.07 \mathrm{eV}\left(610 \mathrm{~cm}^{-1}\right)$ has also been noted; this corresponds to the alreadynoted transition within the ground multiplet.

\subsection{Giant anisotropy}

Though it is not possible to treat the numerous investigations on resonance in various garnets, the position and width of lines, relaxation times etc., we shall make an exception in the case of Dillon's et al.62,89-91. Their particularly spectacular results shed light on the energies of the rare earths as a function of the orientation of the applied field. These authors studied the behaviour of a monocrystal of $Y I G$ containing a rare earth, such as $\mathrm{Tb}$, as an impurity in concentration $0.01 \%$ to $0.19 \%$; the ferromagnetic resonance was investigated at liquid helium temperatures. The resonance

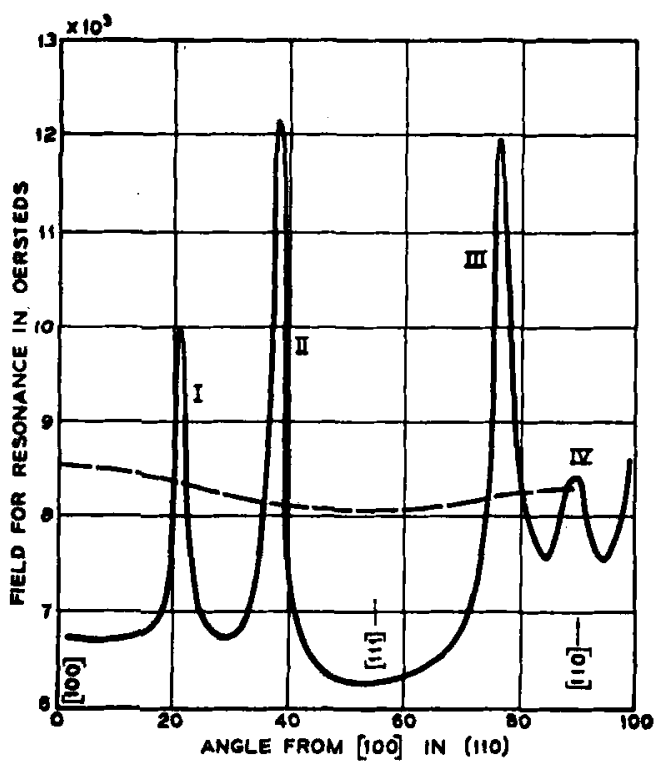

Fig. 15. $H_{\text {rea }}$ in (110) at $1.5^{\circ} \mathrm{K}$ in YIG $(0.2 \% \mathrm{~Tb})$. The frequency was $22989 \mathrm{Mc} / \mathrm{sec}$. The peaks as seen in this plane are designated by Roman numerals. The dashed curve in $H_{\text {res }}$ for the purest YIG with which we have worked. 
is detected, as a function of the orientation of the applied external field relative to the crystal axes. In spite of the small amount of impurities, the resonance field at certain angles varies very sharply (Fig. 15). These peaks have been studied as functions of numerous physical parameters: concen-

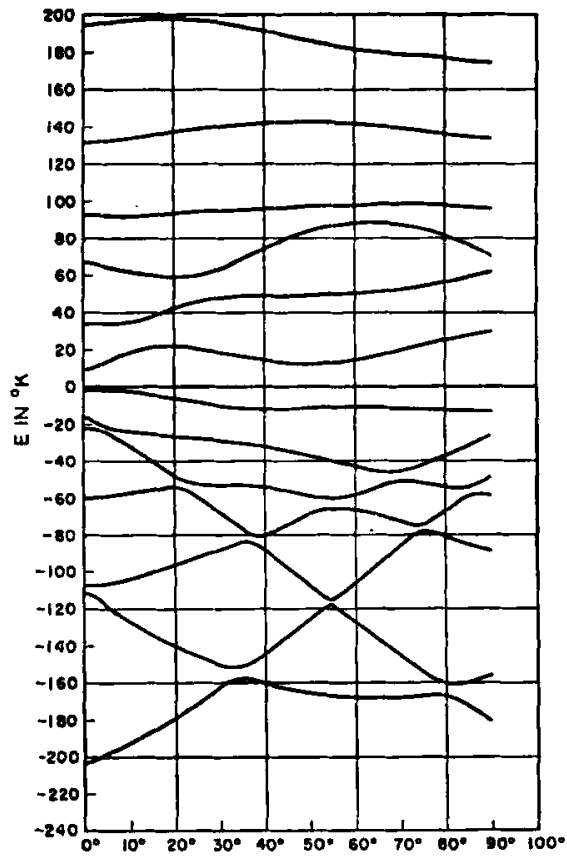

Fig. 16. Energy levels of one site of $\mathrm{Tb}^{+++}$in YIG. The spontaneous iron magnetization is in $(110)$ plane.

tration of $\mathrm{Tb}$, temperature, resonance frequency, etc. The theory which has been given $62,92,93$ shows the fundamental importance of "near-crossing" of levels under the combined influence of crystalline and exchange fields, for certain orientations of the latter. At these near-crossing points, where one level becomes very close to that immediately above it, there is a large curvature of the level. We can describe this by saying that there is both a large magnetic susceptibility and a large anisotropy of the particular level. These two descriptions are in some sense equivalent, and one can study the resonance equations starting from one or the other. If one uses the anisotropy description, the ferromagnetic resonance ${ }^{92,93}$ formula becomes

$$
\omega=\gamma\left[\left(H_{\text {ses }}+H_{\alpha}\right)\left(H_{\text {res }}+H_{\beta}\right)\right]^{\frac{1}{2}} .
$$


$\omega$ is the frequency in the resonance field $H_{\text {res }}$, and $H_{\alpha}$ and $H_{\beta}$ are the effective anisotropy fields for the two principal directions $\alpha, \beta . H_{\alpha}$ is related to the curvature of the level by

$$
H_{\alpha}=\frac{1}{M} \frac{\partial^{2} E}{\partial \theta_{\alpha}^{2}} .
$$

The appearance of a sharp variation in $H_{\alpha, \beta}$, thus explains the observed peaks in $H_{\text {res. }}$.

Dillon and Walker ${ }^{62}$ have tried to explain their results quantitatively by introducing an isotropic exchange field, and a 3-parameter crystalline field for the spherical harmonics of order 2, 4 and 6; each harmonic was chosen to satisfy the electrostatic point-charge model compatible with the crystal structure (Fig. 16). General agreement is good, even quantitatively; unhappily, the results are insensitive to large variations of the four parameters introduced, and it does not seem possible to find the crystalline field easily and accurately in this way.

\subsection{An Example of the "Reconstruction" of a Garnet}

All the parameters obtained on the energy levels, as a function of the orientation of the spontaneous magnetisation, enable us to envisage the calculation of all the microscopic properties starting from these primary parameters. This has been done by Néel and Pauthenet for the simple garnets YIG and $G d I G$, and has been attempted ${ }^{22}$ for the simplest of the remaining garnets, Yb I G. Starting from Wickersheim's results ${ }^{83}$, Henderson and White ${ }^{22}$ calculated the partition function for $\mathrm{Yb}$ I G. They supposed that the magnetisation of the iron ions was temperature independent, which is fully justified below $80^{\circ} \mathrm{K}$. For $M_{\mathrm{s}}$ in the plane 110 , there are only four inequivalent rare earth sites, and optical measurements give the splittings of these four sites. The magnetic partition function was taken as the sum of the partition functions of the individual ions (which we have seen is justified for the optical branches of the spin-wave spectrum). The calculations were performed on an electronic computer, which then enabled an a priori calculation of the free energy as a function of temperature and orientation. One thus sees that the direction of easy magnetisation is [111]. One also deduces the values and thermal variation of the anisotropy constants, $K_{1}$ and $K_{2}$. These values agree with the static measurements of the couple necessary to maintain the magnetisation in a given direction. The saturation magnetisation of a monocrystal varies with the angle, and the magnetic compensation point, found as $7.7^{\circ} \mathrm{K}$, agrees with Pauthenet's 
measurements. The specific heat and the adiabatic cooling of a monocrystal by rotation in an external field were also calculated.

\section{REFERENCES}

1 H. Forestier and G. Guiot-Guillain, C. R. Paris 230, 1844 (1950).

2 G. Guiot-Guillain, C. R. Paris 231, 1832 (1951).

${ }^{3}$ H. Forestier and G. Guiot-Guillain, C. R. Paris 235, 48 (1952); G. Guiot-Guillain, C. R. Paris 237, 1654 (1953).

${ }^{4}$ R. Pauthenet and P. Blum, C. R. Paris 239, 33 (1954).

5 L. Néel, C.R. Paris 239, 8 (1954).

6 G. Guiot-Guillain, R. Pauthenet and H. Forestier, C.R. Paris 239, 155 (1954).

7 S. Geller, Phys. Rev. 99, 1641 (1955).

8 F. Bertaut and F. Forrat, C.R. Paris 242, 382 (1956).

8 R. Pauthenet, C.R. Paris 242, 1859 (1956).

10 R. Pauthenet, C.R. Paris 243, 1499 (1956).

11 R. Aleonard, J. C. Barbier and R. Pauthenet, C.R. Paris 242, 2531 (1956).

12 L. Néel, F. Bertaut, R. Pauthenet and F. Forrat, Comptes Rendus de l'Académie des Sciences d'U.R.S.S. 21, 6 (1957).

13 F. Bertaut and F. Forrat, C.R. Paris 243, 1219 (1956).

14 F. Bertaut and F. Forrat, C.R. Paris 244, 96 (1956).

15 F. Bertaut, F. Forrat, A. Herpin and P. Meriel, C.R. Paris 243, 898 (1956).

16 S. Geller and M. A. Gilleo, Acta Cryst. 10, 243 and 787 (1957).

17 W. P. Wolf and G. P. Rodrigue, J. Appl. Phys. 29, 105 (1958).

18 J. W. Nielsen and E. F. Dearborn, J. Phys. Chem. Solids 5, 202 (1958).

19 J. W. Nielsen, J. Appl. Phys. 31, 51 S (1960).

20 S. Geller and M. A. Gilleo, J. Phys. Chem. Solids 3, 30 (1957).

21 M. A. Gilleo and S. Geller, Phys. Rev. 110, 73 (1958).

22 J. W. Henderson and R. L. White, Phys. Rev. 121, 1627 (1961).

28 R. Aleonard, J. Phys. Chem. Solids 15, 167 (1960).

24 A. Serres, Ann. Phys. (1932).

${ }_{25}$ M. Fallot and P. Maroni, J. Phys. Rad. 12, 256 (1951).

26 E. W. Gorter, Phillips Research Repts 9, 403 (1954).

27 L. Néel, Ann. Phys. 3, 137 (1948).

28 L. Néel, Ann. Phys. 5, 232 (1936).

${ }^{20}$ H. A. Kramers, Physica 1, 182 (1934).

30 P. W. Anderson, Phys. Rev. 79, 350 (1950).

31 Y. Ayant and J. Thomas, C.R. Paris 248, 387 (1959).

32 R. L. White and J. P. Andelin, Phys. Rev. 115, 1435 (1960).

83 W. P. Wolf, Proc. Phys. Soc. 74, 665 (1959).

34 E. Prince, Phys. Rev. 102, 675 (1956).

${ }^{55}$ L. Néel, J. Phys. Rad. 12, 259 (1951).

36 C. Robert, C.R. Paris 251, 2685 (1960).

s7 I. Solomon, C.R. Paris 251, 2675 (1960).

38 W. P. Wolf and J. H. Van Vleck, Phys. Rev. 118, 1492 (1960).

38 R. Pauthenet, J. Phys. Rad. 20, 388 (1959).

40 Y. Ayant and J. Thomas, C.R. Paris 250, 2688 (1960).

41 R. Pappalardo and D. L. Wood, J. Chem. Phys. 33, 1734 (1960).

42 D. Boakes, G. Garton, D. Ryan and W. P. Wolf, Proc. Phys. Soc. 74, 663 (1959).

43 R. F. Pearson and R. W. Teale, Proc. Phys. Soc. 76, 388 (1960).

44 J. H. Van Vleck, Electric and Magnetic Susceptibilities (Oxford, 1932). 
45 G. H. Dieke and L. A. Hall, J. Chem. Phys. 27, 465 (1957).

46 J. H. Van Vleck, J. Phys. Soc. Japan 17, 352 S (1961).

47 V. Jaccarino, B. T. Matthias, M. Peter, H. Suhl and J. H. Wernick, Phys. Rev. Letters 5, 251 (1960).

48 M. A. Gilleo and S. Geller, J. Appl. Phys. 29, 380 (1958).

${ }^{49}$ R. Pauthenet, J. Appl. Phys. 29, 253 (1958).

${ }^{50}$ G. Villers, R. Pauthenet and J. Loriers, J. Phys. Rad. 20, 382 (1959).

${ }_{51}$ G. Goldring, M. Schieber and Z. Vajer, J. Appl. Phys. 31, 205 (1960).

52 A. Aharoni and M. Schieber, J. Phys. Chem. Solids 19, 304 (1961).

53 S. Geller, H. J. Williams and R. C. Sherwood, Phys. Rev. 123, 1692 (1961).

54 W. P. Wolf, J. Appl. Phys. 32, 742 (1961).

65 J. F. Dillon, J. Appl. Phys. 29, 5839 (1958).

56 J. F. Dillon, J. Appl. Phys. 29, 1286 (1958).

${ }^{57}$ J. F. Dillon, J. Phys. Rad. 20, 374 (1959).

58 Y. Ayant and J. Rosset, Ann. Inst. Fourier, Grenoble 10, 459 (1960).

59 R. Pappalardo, J. Chem. Phys. 34, 1380 (1961).

${ }^{60}$ R. Pappalardo, J. Chem. Phys. 31, 1050 (1959).

${ }^{61}$ Y. Ayant and J. Thomas, C.R. Paris 248, 1955 (1959).

62 J. F. Dillon Jr. and L. R. Walker, Phys. Rev. 124, 1401 (1961).

63 D. T. Edmonds and R. G. Pekisen, Phys. Rev. Letters 2, 499 (1959).

64 J. E. Kunzier, L. R. Walker and J. K. Galt, Phys. Rev. 119, 1609 (1960).

65 S. S. Shinozaki, Phys. Rev. 122, 388 (1961).

68 A. B. Harris and H. Meyer, Phys. Rev. 127, 101 (1962).

67 R. L. Douglass, Phys. Rev. 120, 1612 (1960).

68 R. C. Le Craw and R. L. Walker, J. Appl. Phys. 32, 167 S (1959).

${ }^{B \theta}$ E. H. Turner, Phys. Rev. Letters 5, 100 (1960).

70 B. Dreyfus, J. Phys. Chem. Solids 23, 287 (1962).

71 M. Tinkham, Phys. Rev. 124, 311 (1961).

72 A. B. Harrris, private communication.

78 A. J. Sievers and M. Tinkham, Phys. Rev. 124, 321 (1961).

74 A. J. Sievers and M. Tinkham, Phys. Rev. 129, 1995 (1963).

75 B. Lüthi, J. Phys. Chem. Solids 23, 35 (1962).

${ }^{76}$ R. L. Douglass, Phys. Rev. 129, 1132 (1963).

77 H. Sato, Progr. Theoret. Phys. (Kyoto) 13, 119 (1955).

78 V. G. Bar'yakhtar and G. I. Urushadze, Soviet Phys. JETP 7, 875 (1958).

70 K. A. Wickersheim, Lefever and Hanking, J. Chem. Phys. 32, 271 (1960).

80 J. F. Dillon, J., Appl. Phys. 29, 539 (1958).

81 A. M. Clogston, J. Appl. Phys. 31, 198 S (1960).

82 K. A. Wickersheim and R. L. White, Phys. Rev. Letters 4, 123 (1960).

$88 \mathrm{~K}$, A. Wickersheim, Phys. Rev, 122, 1376 (1961).

84 J. W. Carson and R. L. White, J. Appl. Phys. 31, 535 (1960).

85 B. Dreyfus, J. Verdone-Thuilier and M. Veyssie-Counillon, C.R. Paris 252, 1928 (1961) and 256, 646 (1963).

$86 \mathrm{~J}$. Thomas, private communication.

87 J. Kaplan and C. Kittel, J. Chem. Phys. 21, 760 (1953).

88 H. Watanabe and B. N. Brockhouse, Phys. Rev, 128, 67 (1962).

89 J. F. Dillon Jr., Phys. Rev. 111, 1476 (1958).

90 J. F. Dillon Jr. and J. W. Nielsen, Phys. Rev. 120, 105 (1960).

91 J. F. Dillon Jr., Phys. Rev. 127, 1495 (1962).

${ }^{82}$ C. Kittel, Phys. Rev. Letters 3, 169 (1959).

98 C. Kittel, Phys. Rev, 117, 681 (1960). 